University of Nebraska - Lincoln

DigitalCommons@University of Nebraska - Lincoln

Faculty Publications, Department of Mathematics

Mathematics, Department of

3-2009

\title{
Parameterizing the growth-decline boundary for uncertain population projection models
}

\author{
Joan Lubben \\ University of Nebraska-Lincoln, jolubben@dwu.edu \\ Derek Boeckner \\ University of Nebraska - Lincoln \\ Richard Rebarber \\ University of Nebraska - Lincoln, rrebarber1@unl.edu \\ Stuart Townley \\ University of Exeter, UK \\ Brigitte Tenhumberg \\ University of Nebraska - Lincoln, btenhumberg2@unl.edu
}

Follow this and additional works at: https://digitalcommons.unl.edu/mathfacpub

Part of the Mathematics Commons

Lubben, Joan; Boeckner, Derek; Rebarber, Richard; Townley, Stuart; and Tenhumberg, Brigitte, "Parameterizing the growth-decline boundary for uncertain population projection models" (2009). Faculty Publications, Department of Mathematics. 51.

https://digitalcommons.unl.edu/mathfacpub/51

This Article is brought to you for free and open access by the Mathematics, Department of at DigitalCommons@University of Nebraska - Lincoln. It has been accepted for inclusion in Faculty Publications, Department of Mathematics by an authorized administrator of DigitalCommons@University of Nebraska - Lincoln. 


\title{
Parameterizing the growth-decline boundary for uncertain population projection models
}

\author{
Joan Lubben, ${ }^{1}$ Derek Boeckner, ${ }^{1}$ Richard Rebarber, ${ }^{1}$ Stuart Townley, ${ }^{2}$ and Brigitte Tenhumberg ${ }^{1,3}$ \\ 1. Department of Mathematics, University of Nebraska-Lincoln, Lincoln, NE 68588-0130, USA \\ 2. Mathematics Research Institute, School of Engineering, Computing and Mathematics, University of Exeter, Exeter, EX44QF, UK \\ 3. School of Biological Sciences, University of Nebraska-Lincoln, Lincoln, NE 68588-0118, USA \\ Corresponding author - R. Rebarber, Department of Mathematics, University of Nebraska-Lincoln, \\ 203 Avery Hall, Lincoln, NE 68588-0130, USA; email rrebarber1@unl.edu
}

\begin{abstract}
We consider discrete time linear population models of the form $\mathbf{n}(t+1)=\mathbf{A n}(t)$ where $\mathbf{A}$ is a population projection matrix or integral projection operator, and represents a structured population at time $t$. It is well known that the asymptotic growth or decay rate of $\mathbf{n}(t)$ is determined by the leading eigenvalue of $\mathbf{A}$.

In practice, population models have substantial parameter uncertainty, and it might be difficult to quantify the effect of this uncertainty on the leading eigenvalue. For a large class of matrices and integral operators $\mathbf{A}$, we give sufficient conditions for an eigenvalue to be the leading eigenvalue.

By preselecting the leading eigenvalue to be equal to 1, this allows us to easily identify, which combination of parameters, within the confines of their uncertainty, lead to asymptotic growth, and which lead to asymptotic decay. We then apply these results to the analysis of uncertainty in both a matrix model and an integral model for a population of thistles. We show these results can be generalized to any preselected leading eigenvalue.
\end{abstract}

Keywords: population projection matrix, integral projection model, robustness, asymptotic growth rate

\section{Introduction}

Projection models are commonly used for predicting the dynamics of structured populations (e.g., Caswell, 2001; Ehrlén, 2000; Mandujano et al., 2001; Seno and Nakajima, 1999). If there are finitely many stages, or if the stages are determined by discretizing a continuous variable (such as size), matrix projection models are used. To avoid such a discretization, integral projection models (Easterling et al., 2000; Ellner and Rees, 2006) can be used. Both of these modeling approaches require multiple life-history parameters, and the data to accurately estimate those parameters is often lacking; as a result of insufficient data these models suffer from high parameter uncertainty. Furthermore, parameters often vary on a spatial and temporal scale, and stochastic models are even more data hungry (Doak et al., 2005).

There are well-established methods for local perturbation analysis, such as elasticity and sensitivity of matrix transition rates or parameter values (Caswell, 2001), which examine the consequences of very small perturbations of single, independent parameters (Caswell, 2001). When calculating elasticities only one matrix element or life history parameter is varied, while others remain the same, so using elasticity only gives us information about varying one parameter. In the case of multiple perturbations, elasticities can only be used directly if the transition probabilities are changed by the same proportion
(Mills et al., 1999). Biological limits may constrain how much the transition rate with the highest elasticity can be changed, so changes in a transition rate with a lower elasticity may be required to achieve the management goal (Lubben et al., 2008). Furthermore, elasticity analyses does not take into account uncertainty in the data. Parameter uncertainty has been incorporated into elasticity analysis by incorporating standard deviation into the definition of elasticity (Ehrlén and van Groenendael, 1998), including covariation between parameters (van Tienderen, 1995), and by adding random components to the parameters (Wisdom and Mills, 1997). Caswell (2000) and de Kroon et al. (2000) both give in-depth discussions of the caveats for some of these methods.

Global perturbation analysis is used when relatively large changes in parameters values are being considered. Large uncertainties in the parameter values often occur when the sample size is small. Management actions may also change parameter values by large amounts. Demographic and environmental stochasticity lead to mean parameter values which vary over space and time. Using sensitivity and elasticity analysis to infer the effect of large perturbations on the asymptotic population growth rate $\lambda$ can result in misleading conclusions (Deines et al., 2007; Hodgson and Townley, 2004; Hodgson et al., 2006; Mills et al., 1999; Tenhumberg et al., 2008). One possible approach to global perturbation analysis of matrix models is Monte Carlo analysis (Tenhumberg et al., 2008). 
In this paper, we present an analytical alternative for matrix and integral projection models. We parameterize the growthdecline boundary in terms of the most significant parameters. We can use this to determine how robust $\lambda$ is within the confines of the uncertainty in the parameters. This can be applied to models for endangered (or invasive) species. Sensitivity and elasticity tells us what management strategy we should focus on in order to increase (or decrease) the asymptotic growth rate. Our method tells us whether a specific management strategy can achieve the desired population growth rate (e.g. $\lambda>1$ ) in the face of both parameter and stochastic uncertainty.

The starting point for the analysis is the identification of a "nominal point" in the multidimensional parameter space. The nominal point uses our best estimates for the parameters, or our best guess in the case of poorly known parameters. We are interested in the effects of perturbations away from the nominal point on relevant system properties. In this paper the system property we are interested in is the asymptotic growth rate $\lambda$, but the approach could be modified for other easily quantifiable system properties. In our approach we first calculate the hypersurface representing population stasis $(\lambda=1)$; then one side of the surface indicates the parameter space for a growing population $(\lambda>1)$, and the other side for a declining population $(\lambda<1)$. In case of an endangered species the nominal point will be on the declining side, while the nominal point for an invasive species will be on the growing side. In both cases it is important to know how sensitive the qualitative predictions are to parameter perturbations, especially when a predicted growing population will decline or a predicted declining population will grow. This is relevant for population management, i.e. choosing a strategy that works under a range of likely environmental conditions (including temporal and or spatial variation of population parameters), or for understanding how reliably ecological factors such as predation or competition can limit population growth. The distance from the nominal point to the hypersurface representing stasis is a measure of the robustness of the stability (or lack of stability) of the system. The larger the distance, the more robust the model predictions are to parameter perturbations, i.e. the less likely that perturbations cause a qualitative change in model predictions (e.g., achieving or not achieving a desired management goal). The distance can be evaluated numerically, or it can be evaluated graphically when there are two or three relevant parameters.

The boundary between growth and decline is characterized by the largest (in modulus) eigenvalue of the projection matrix or operator, amongst its totality of eigenvalues. It is not always easy to determine whether an eigenvalue identified in computations is actually the leading eigenvalue $\lambda$. To illustrate this difficulty in the simplest situation, consider the case of a general, non-negative $2 \times 2$ matrix

$$
\mathbf{A}=\left(\begin{array}{ll}
a & b \\
c & d
\end{array}\right)
$$

A has characteristic polynomial

$$
\operatorname{det}(s \mathbf{I}-\mathbf{A})=s 2-(a+d) s+a d-b c
$$

with eigenvalues

$$
\lambda_{ \pm}=1 / 2\left(a+d \pm \sqrt{(a-d)^{2}+4 b c}\right)
$$

From this it follows that $\lambda=\rho$ is an eigenvalue of $\mathbf{A}$ if

$$
\rho^{2}-(a+d) \rho+a d-b c=0,
$$

while $\rho$ is the dominant eigenvalue of $\mathbf{A}$ if

$$
\lambda_{+}=1 / 2\left(a+d \pm \sqrt{(a-d)^{2}+4 b c}\right)=\rho
$$

Even in this $2 \times 2$ case, it is clear that requiring $\rho$ to be an eigenvalue of $\mathbf{A}$ is analytically and computationally simpler than requiring $\rho$ to be the dominant eigenvalue of $\mathbf{A}$. In higher dimensional cases, and moreover in the case of integral projection operators, this claim will be much stronger. On the other hand, if we can already establish that $\mathbf{A}$ has only one eigenvalue $\lambda \geq \rho$, then $\lambda=\rho$ is the dominant eigenvalue if, and only if, $\operatorname{det}(\rho \mathbf{I}-\mathbf{A})=0$. Therefore, we would ideally like to find simple conditions which guarantee that an eigenvalue is the dominant eigenvalue.

This issue of identifying the growth-decline boundary arose in a robustness study of a $3 \times 3$ matrix model of peregrine falcons (Deines et al., 2007). In this paper, the proof showing that we had indeed identified the "correct" eigenvalue was specific to the model, although we could demonstrate numerically that the method worked for other matrices. In this paper we provide an analytical proof that the method can be applied to virtually all ecologically relevant density-independent single species population matrix models (irrespective of the number of age/stage classes). It can be used as global perturbation analysis of matrix elements as well as lower level parameters (parameters that are used to calculate matrix elements). Additionally we extend the proof to include the analysis of a large class of integral projection models. We illustrate this approach with two different weedy thistle species: Cirsium vulgare (matrix model, Tenhumberg et al., 2008) and Onopordum illyricum (integral projection model, Ellner and Rees, 2006).

C. vulgare is a late-season flowering, tap-rooted, short-lived perennial plant. The juvenile rosette phase typically lasts for one to several years prior to the single flowering episode after which they die (monocarpic plant, Guretzky and Louda, 1997). One of the key factors controlling this species in western tallgrass prairie in eastern Nebraska, USA, is native floralfeeding insects; most of the seed reduction can be attributed to destruction of floral meristems by moth larvae (Pyralidae, Pterophoridae) and receptacles, florets, and developing seeds by the moths and by picture-winged flies (Tephritidae) (Louda and Rand, 2003). Additionally, weed management practices likely affect its demography in rural areas. Roadside vegetation is generally mowed early and late in the growing season, and intensive row-crop agriculture involves cultivation and herbicide application.

O. illyricum is also a monocarpic perennial across its entire current range (Pettit et al., 1996). It flourishes in fertile soils and is adapted to warmer climate with dry summers (Briese et al., 2002). Reproduction only occurs by seed, and seeds remain viable in the seed bank for many years (Goss, 1924). In its native range $O$. illyricum is attacked by a large variety of insect species (129 insect species feed on Onopordum spp. in Europe, (Briese et al., 1994)), but in its introduced range like Australia, insect herbivores play a minor role in O. illyricum population dynamics. This thistle became a noxious weed in Australia after widespread pasture improvements (fertilization) and is mainly limited by microsite availability (Groves et al., 1990).

\section{Methods}

In this paper, we consider two important classes of population models - Population Projection Matrix Models (PPM) and Integral Projection Models (IPM). The latter are not as familiar as the former, and require some more mathematical analysis (mostly presented in the Appendix B), so we will emphasize the similarities. 
Population projection matrix models: In these systems the population vector consists of finitely many discrete stages. If there are $S$ stages, the population vector $\mathbf{n}$ is in $\mathbb{R}^{S}$, with all entries nonnegative. If the population vector during year $t$ is denoted $\mathbf{n}(\mathrm{t})$, and if $\mathbf{A}$ is the $S$ by $S$ projection matrix for this population, then $(\mathbf{n}(t))_{t=0}^{\infty}$ satisfies the discrete time dynamical system

$$
\mathbf{n}(t+1)=\mathbf{A n}(t)
$$

The total population is

$$
\|\mathbf{n}\|:=n_{1}+n_{2}+\ldots n_{s}
$$

the 1-norm of $\mathbf{n}$.

The long term growth rate for solutions of (2.1) is determined by the eigenvalue or eigenvalues of $\mathbf{A}$ of maximum modulus. For a matrix $\mathbf{M}$, when there is only one eigenvalue of maximum modulus, we call it the leading eigenvalue, and refer to it as $\lambda(\mathbf{M})$. The spectral radius, $r(\mathbf{M})$, is the largest modulus of an eigenvalue of $\mathbf{M}$. If $r(\mathbf{A})>1$ (or $r(\mathbf{A})<1$ ), then the total population increases (or decreases) geometrically, i.e. there exists $m>0$ and $\rho>1$ (or $0<\rho<1$ ) such that

$$
\|\mathbf{n}(k)\| \geq m \rho^{k},\left(\text { or }\|\mathbf{n}(k)\| \leq m \rho^{k}\right) k=0,1, \ldots
$$

The following is a well-known consequence of the PerronFrobenius theorem. We state it as a Proposition for future reference. A primitive matrix is a nonnegative square matrix $\mathbf{A}$ such that $\mathbf{A}^{k}>0$ for some positive integer $k$.

\section{Proposition}

Suppose that $\boldsymbol{A}$ is primitive. Then $\boldsymbol{A}$ has a leading eigenvalue $\lambda(\mathbf{A})$ $=r(\mathbf{A})>0$ with associated right dominant eigenvector $\mathbf{v}>0$, the socalled Perron vector of $\mathbf{A}$. The Perron vector is the only positive eigenvector of $\mathbf{A}$ and can be normalized so that $\|v\|_{1}=1$. Moreover, the system (2.1) has $\mathbf{v}$ as an asymptotic stable population distribution, i.e.

$$
\lim _{k \rightarrow \infty} \mathbf{n}(k) /\|\mathbf{n}(k)\|=\mathbf{v} .
$$

Integral projection models: A class of integral population projection operators is introduced in Easterling (1998), Easterling et al. (2000), and Ellner and Rees (2006). Let $n(x, t)$ be the population distribution as a function of the stage $x$ at time $t$. For example, $x$ could be the size of the individual, with maximal size $M_{s}$. We discuss this case in more detail before turning to the general case. The role of the matrix is replaced by an integral operator with projection kernel $k(y, x)$, yielding the integro-difference equation

$$
n(y, t+1)=\int_{0}^{M_{s}} k(y, x) n(x, t) d x
$$

In particular, the kernel determines how the distribution of stage $x$ individuals at time $t$ moves to the distribution of stage $y$ individuals at time $t+1$, much the same way that the $(i, j)$ th entry of a projection matrix determines how an individual in stage $j$ at time $t$ moves to state $i$ at time $t+1$.

Let $\Omega$ be the set of possible stages $y$. For instance, if $y$ is size, $\Omega$ is the interval $\left[0, M_{s}\right]$, where $M_{s}$ is the maximum size. We can write (2.3) as

$$
n(y, t+1)=\int_{\Omega} k(y, x) n(x, t) d x .
$$

The stage variable $y$ does not have to be a scalar. In an example in the next section, originally given in Ellner and Rees (2006), $\Omega$ is the set of all size-age pairs, where age is measured discretely with maximum age $M_{a^{\prime}}$ so $\Omega=\left\{(x, a) \mid x \in\left[0, M_{s}\right], a \in\right.$ $\left.\left\{0,1,2, \ldots M_{a}\right\}\right\}$. Then the projection model is still given by (2.4), but if $\Omega$ is not a subset of $\mathbb{R}, d y$ will indicate integration with respect to a measure; see Section 3, and Ellner and Rees (2006), for a discussion of this.

Integral equations such as (2.4) can be analyzed in much the same way as matrix-based models of the form (2.1). The Banach space $L_{1}(\Omega)$ is defined to be the set of functions mapping $\Omega$ to $\mathbb{R}$ which are "measurable" (which is implied by continuity) and whose 1-norm, given by

$$
\|\mathbf{v}\|:=\int_{\Omega}|\mathbf{v}(x)| d x,
$$

is less than infinity. Since this norm can be interpreted as the total population, $L_{1}(\Omega)$ is analogous to $\mathbb{R}^{S}$ with norm given in (2.2). For a population function $n(x, t)$, it is sometimes useful to distinguish between the function $n(x, t)$ of two variables and the vector $\mathbf{n}(t)=n(\cdot, t)$ which is in $L_{1}(\Omega)$ for a given $t$. Define the operator $\mathbf{A}: L_{1}(\Omega) \rightarrow L_{1}(\Omega)$ by

$$
(\operatorname{Av})(\cdot):=\int_{\Omega} k(\cdot, x) \mathbf{v}(x) d x
$$

Then the Equation (2.4) is equivalent to (2.1).

Easterling (1998), Easterling et al. (2000), and Ellner and Rees (2006) show that for a class of kernels $k$, the solution of (2.4) satisfies the conclusions of Proposition 2.1; see also Appendix $B$ below.

We will denote matrices and integral operators by bold capital letters, such as A. We will denote vectors, $S$ by 1 matrices (i.e. column vectors) and $L_{1}(\Omega)$ functions by bold lower case letters, such as $\mathbf{d}$. We will denote 1 by $S$ matrices (i.e. row vectors) and functionals on $L_{1}(\Omega)$ by bold lower case letter with a $T$ superscript, to denote transpose, such as $\mathbf{e}^{T}$.

A key modelling and analysis issue is that the matrix or operator will involve parameters, for instance fecundity or survival parameters. Usually these parameters will be uncertain. These uncertainties in A are typically structured, that is, the uncertainties occur only in specific locations in the model. For example, in a Leslie matrix, it only makes biological sense to perturb the top row and/or the sub-diagonals. These uncertainties can be described by $m$ parameters $\left(p_{1}, p_{2}, \ldots, p_{m}\right)$. When $\mathbf{A}$ is a matrix, we can typically choose $m \leq S^{2}$, the number of entries.

In this paper, we are interested in the effect of the uncertainties on the asymptotic growth rate $\lambda$. We can denote the explicit dependence of $\mathbf{A}$ and $\lambda$ on $\left(p_{1}, p_{2}, \ldots, p_{m}\right)$ by writing

$$
\mathbf{A}=\mathbf{A}\left(p_{1}, p_{2}, \ldots, p_{m}\right), \quad \lambda=\lambda\left(p_{1}, p_{2}, \ldots, p_{m}\right) \text {. }
$$

We identify a set $\mathcal{P}$ of admissible parameters as those $\left(p_{1}, p_{2}, \ldots\right.$, $\left.p_{m}\right)$ which make biological sense in the model, and if necessary, are such that $\mathbf{A}\left(p_{1}, p_{2}, \ldots, p_{m}\right)$ has desirable mathematical properties, defined below. We can now describe one way of analyzing the effect of changes in the parameters on $\lambda$.

Consider the subset of $\mathcal{P}$ given by

$$
C:=\left\{\left(p_{1}, p_{2}, \ldots, p_{m}\right) \in \mathcal{P} \mid \lambda\left(p_{1}, p_{2}, \ldots, p_{m}\right)=1\right\} .
$$

This is the set of $\left(p_{1}, p_{2}, \ldots, p_{m}\right)$ for which the leading eigenvalue $\lambda(\mathbf{A})=1$. Mathematically, this set is a hypersurface. If we are considering two uncertain parameters, then $m=2$ and $C$ is a curve. If we are considering three uncertain parameters, then $m=3$ and $C$ is an ordinary surface (that is, a two dimensional object in three dimensions).

If we are concerned with maintaining a particular growth rate, say $3 \%$, then we would replace $C$ by $C_{1.03}$, where for arbitrary $\mu \in \mathbb{R}$,

$$
C_{\mu}:=\left\{\left(p_{1}, p_{2}, \ldots, p_{m}\right) \in \mathcal{P} \mid \lambda\left(p_{1}, p_{2}, \ldots, p_{m}\right)=\mu\right\} .
$$

In some applications we will be interested in identifying the set of all parameters which lead to asymptotic growth:

$$
C_{+}:=\left\{\left(p_{1}, p_{2}, \ldots, p_{m}\right) \in \mathcal{P} \mid \lambda\left(p_{1}, p_{2}, \ldots, p_{m}\right)>1\right\} .
$$


For other applications, we will be interested in identifying the set of all parameters which lead to asymptotic decay:

$$
C_{-}:=\left\{\left(p_{1}, p_{2}, \ldots, p_{m}\right) \in \mathcal{P} \mid \lambda\left(p_{1}, p_{2}, \ldots, p_{m}\right)<1\right\}
$$

When the notion of "side" is made precise mathematically, we can prove that $C_{+}$is one side of the hypersurface $C$ and $C_{-}$is on the other side of $C$. This is done in Appendix $C$.

Our primary interest in this paper is in finding a usable formula for the hypersurface $C$. We assume that in applications we have nominal values for the parameters $\left(p_{1}, p_{2}, \ldots, p_{m}\right)$, that is, those values that are determined by experiment or some other method. We denote these nominal values by the point $\mathbf{q}_{0}$ in $\mathbf{R}^{m}$. We also assume that it is either considered "desirable" for the population to be in asymptotic decline (for instance, for an invasive species), or "desirable" for the population to be asymptotically increasing (for instance, for an endangered species).

The following robustness questions can be addressed once we have a formula for $C$ :

- If $\lambda\left(\mathbf{q}_{0}\right)>1$, and it is good for the population to asymptotically increase, then we are interested in how much the nominal parameters can be perturbed before population growth is lost.

- If $\lambda\left(\mathbf{q}_{0}\right)<1$, and it is good for the population to asymptotically decrease, then we are interested in how much the nominal parameters can be perturbed before population decay is lost.

The following control, i.e. population management, questions can be addressed once we have a formula for $C$ :

- If $\lambda\left(\mathbf{q}_{0}\right)>1$, and it is good for the population to asymptotically decrease, then we are interested in how much the parameters have to be perturbed before population decay is achieved.

- If $\lambda\left(\mathbf{q}_{0}\right)<1$, and it is good for the population to asymptotically increase, then we are interested in how much the parameters have to be perturbed before population growth is achieved.

However, we still need to find a usable equation for $C$. The obvious starting point is to consider the hypersurface $\Gamma$ on which at least one eigenvalue of $\mathbf{A}$ is 1 :

$\Gamma=\left\{\left(p_{1}, p_{2}, \ldots, p_{m}\right) \in \mathcal{P} \mid 1\right.$ is an eigenvalue of $\left.\mathbf{A}\left(p_{1}, p_{2}, \ldots, p_{m}\right)\right\}$.

In the matrix case, the hypersurface $\Gamma$ is obtained from inspecting the characteristic polynomial: letting I denote the $n \times n$ identity matrix,

$\Gamma:=\left\{\left(p_{1}, p_{2}, \ldots, p_{m}\right) \in \mathcal{P} \mid \operatorname{det}\left(\mathbf{I}-\mathbf{A}\left(p_{1}, p_{2}, \ldots, p_{m}\right)\right)=0\right\}$.

More generally, we can let $\Gamma_{\mu}$ be the hypersurface on which at least one eigenvalue of $\mathbf{A}$ is $\mu$ :

$\Gamma_{\mu}=\left\{\left(p_{1}, p_{2}, \ldots, p_{m}\right) \in \mathcal{P} \mid \mu\right.$ is an eigenvalue of $\left.\mathbf{A}\left(p_{1}, p_{2}, \ldots, p_{m}\right)\right\}$. for matrices this is

$\Gamma_{\mu}:=\left\{\left(p_{1}, p_{2}, \ldots, p_{m}\right) \in \mathcal{P} \mid \operatorname{det}\left(\mu \mathbf{I}-\mathbf{A}\left(p_{1}, p_{2}, \ldots, p_{m}\right)\right)=0\right\}$.

If $\mathbf{A}$ is a matrix, it is easy to find a formula for $\Gamma$, and if $\mathbf{A}$ is an integral operator, it is often relatively easy to approximate $\Gamma$. However, as alluded to above, there is no guarantee that $\Gamma$ is the same as $C$ because we cannot guarantee a priori that we have not just found a sub-dominant eigenvalue of $\mathbf{A}\left(p_{1}, p_{2}, \ldots\right.$, $\left.p_{m}\right)$. We saw this even in the simple $2 \times 2$ case discussed in the Introduction. Therefore it would be useful to have conditions under which $C_{\mu}=\Gamma_{\mu}$. In Deines et al. (2007) this is shown for a particular matrix example, using linear algebra techniques that are specific to the system in that paper. Here, we develop a systematic approach which shows that $\Gamma$ is, in very natural situations, the same as $C$. We first discuss these results, first for PPMs, and then for IPMs.

\subsection{The leading eigenvalue of a PPM}

It is well-known that a Leslie matrix has only one positive real eigenvalue. Anticipating the result below, this result follows from our results because a Leslie matrix is simply a rank one, non-negative perturbation of a nilpotent non-negative matrix.

We now state our main mathematical result, which generalizes this familiar result for Leslie matrices to a wide class of matrices which arise naturally in population projection models. We then explain how it is used in population problems.

Theorem 2.2. Suppose that $\mathbf{A}$ is a primitive matrix and can be written as $\mathbf{A}=\mathbf{A}_{0}+\mathbf{d e}^{T}$ where

1. $\mathbf{A}_{0}$ is a nonnegative matrix;

2. $\mathbf{d}$ is a column vector, $\mathbf{e}^{T}$ is a row vector, and at least one of them is nonnegative;

If $\lambda>r\left(\mathbf{A}_{0}\right)$ is an eigenvalue of $\mathbf{A}$, then $\lambda=\lambda(\mathbf{A})$.

In Appendix $\mathrm{A}$ we give a proof of this theorem, show why it is a generalization of the result for Leslie matrices, and illustrate how it is typical that a PPM will be of the form in the hypotheses of this Theorem.

We now return to the problem addressed in the Introduction. Suppose we are interested in identifying the hypersurface (2.5). We can be now more precise about the set $\mathcal{P}$ of admissible perturbations: Let $\mathcal{P}$ be the set of all $\left(p_{1}, p_{2}, \ldots, p_{m}\right) \in$ $\mathbb{R}^{m}$ such that $\mathbf{A}\left(p_{1}, p_{2}, \ldots, p_{m}\right)$ is of the form $\mathbf{A}_{0}+\mathbf{d e} \mathbf{e}^{T}$ satisfying the hypotheses of Theorem 2.2 with $r\left(\mathbf{A}_{0}\right)<1$.

This might seem like an awkward mathematical definition, but is it very natural. Most PPM are primitive and can be written as a survival matrix $\mathbf{A}_{0}$ plus a fecundity matrix, where the survival matrix $\mathbf{A}_{0}$ is nonnegative and column sub-stochastic (i.e. the columns sum to $<1$ ) and the fecundity matrix is zero everywhere except for the first row. This shows that $r\left(\mathbf{A}_{0}\right)<1$ and the fecundity matrix can be decomposed into the form $\mathrm{de}^{T}$. Hence $\mathcal{P}$ includes all parameters for which $\mathbf{A}\left(p_{1}, p_{2}, \ldots, p_{m}\right)$ is this very common type of PPM. This is illustrated in the Results section.

Corollary 2.3. For $\mu \geq 1, C_{\mu}=\Gamma_{\mu}$.

Proof. $\Gamma_{\mu}$ is the set of all $\left(p_{1}, p_{2}, \ldots, p_{m}\right) \in \mathcal{P}$ such that some eigenvalue of $\mathbf{A}$ is $\mu$. From Theorem 2.2, if some eigenvalue of $\mathbf{A}\left(p_{1}, p_{2}, \ldots, p_{m}\right)$ is $\mu$, then the leading eigenvalue of $\mathbf{A}\left(p_{1}, p_{2}, \ldots\right.$, $\left.p_{m}\right)$ is $\mu$, so $\Gamma_{\mu}$ must be the same as the set of all $\left(p_{1}, p_{2}, \ldots, p_{m}\right)$ $\in \mathcal{P}$ such that the leading eigenvalue is $\mu$. Hence $\Gamma_{\mu}=C_{\mu}$. 000

Remark 2.4. We can modify the above results in the case where $\mathcal{P}$ is the set of all parameters $\left(p_{1}, p_{2}, \ldots, p_{m}\right)$ such that $\mathbf{A}\left(p_{1}, p_{2}, \ldots, p_{m}\right)$ has the form $\mathbf{A}_{0}+\mathbf{d e}^{T}$ satisfying the hypotheses of Theorem 2.2 with $r\left(\mathbf{A}_{0}\right)<\eta$ for some $\eta>0$. Then Corollary 2.3 would be true for $\mu \geq \eta$.

From Corollary 2.3 we see that for a large class of PPMs, we can find $C_{\mu}$ by finding $\Gamma_{\mu}$. This is illustrated in the Results section.

Also of interest to ecologists would be the shortest distance from the nominal values to $C_{\mu}$. We show in Appendix D how to plot the curve, $C_{1}$, and how to calculate the closest point on the $C_{1}$ curve to the nominal values for our example in the Results section.

\subsection{The leading eigenvalue of an IPM}

In Appendix B we discuss a class of integral operators, introduced in Ellner and Rees (2006), of the form (2.4), for which there is an analog of the Perron-Frobenius Theorem. In particular, conditions on A are given in Theorem B.6 which guarantee that (2.1) satisfies the conclusion of Proposition 2.1. These conditions are that $\mathbf{A}$ is positive, compact, and $u$-bounded. These three concepts are defined in Appendix B. 
As in the PPM case, an IPM operator A can usually be decomposed into the sum of a survival operator $\mathbf{A}_{0}$ and a fecundity operator $\mathbf{F}$. Furthermore, $\mathbf{F}$ can often be decomposed into a product $\mathbf{d e}^{T}$ : if $\mathbf{v}$ is a population vector, $\mathbf{e}^{T} \mathbf{v}$ is the total number of offspring from all states, and $\mathbf{d}$ distributes these offspring into the states. Therefore, $\mathbf{A}=\mathbf{A}_{0}+\mathbf{d e} \mathbf{e}^{T}$. Roughly speaking, any single-species where the state of the offspring is independent of the parents' state can be written in this manner. For instance, in a plant model, the seeds are the same independent of the size of the plant that produced them.

If $\mathbf{A}$ is a positive, compact and $u$-bounded operator, then there are results for $\mathbf{A}$ which are analogous to Theorem 2.2 and Corollary 2.3. We assume a cone in the Banach space $\mathcal{B}$, has been defined, thus determining a definition of "positive": see Definitions B.1-B.4 in Appendix B.

Theorem 2.5. Suppose that $\mathbf{A}$ is positive, compact and $u$-bounded and can be written as $\mathbf{A}=\mathbf{A}_{0}+\mathbf{d e}^{T}$ where

1. $\mathbf{A}_{0}: \mathcal{B} \rightarrow \mathcal{B}$ is a non-negative linear operator;

2. $\mathbf{d}: \mathbb{R} \rightarrow \mathcal{B}, \mathbf{e}^{T}: \mathcal{B} \rightarrow \mathbb{R}$, and at least one of them is positive; If $\lambda>r\left(\mathbf{A}_{0}\right)$ is an eigenvalue of $\mathbf{A}$, then $\lambda=\lambda(\mathbf{A})$.

The proof of this theorem is in Appendix B.

Now suppose $\mathbf{A}=\mathbf{A}\left(p_{1}, p_{2}, \ldots, p_{m}\right)$. We now can identify the set of admissible parameters as those $\left(p_{1}, p_{2}, \ldots, p_{m}\right)$ such that $\mathbf{A}\left(p_{1}, p_{2}, \ldots, p_{m}\right)$ satisfy the hypotheses of Theorem 2.5 . It follows immediately that Corollary 2.3 is also true for $\mathbf{A}$ which satisfy the hypotheses of Theorem 2.5. Remark 2.4 also holds for these IPM.

\section{Results}

\subsection{A PPM thistle model}

We consider a model for an invasive thistle with 19 parameters $a$ through $s$, see Tenhumberg et al. (2008). This model is of the form (2.1), with

$\mathbf{A}=\left[\begin{array}{llll}0 & 0 & {[\ln q(1-h) m o](1-g)} & {[\ln q(1-h)](1-g)} \\ s c f & 0 & {[\ln q(1-h) m o](g s f)} & {[\ln q(1-h)](g s f)} \\ 0 & a d r & b d r j(1-\operatorname{lm}) & 0 \\ 0 & (1-a) \operatorname{epi} i & (1-b) \operatorname{epj}(1-\operatorname{lm}) & \operatorname{epk}(1-l)\end{array}\right]$

Letting $s_{i j}$ represent non-fecundity entries, and $c_{1}=(1-g)$, $c_{2}=g s f, f_{3}=\ln q(1-h) m o, f_{4}=\ln q(1-h)$, we obtain

$\mathbf{A}=\left[\begin{array}{llll}0 & 0 & c_{1} f_{3} & c_{1} f_{4} \\ s_{21} & 0 & c_{2} f_{3} & c_{2} f_{4} \\ 0 & s_{32} & s_{33} & 0 \\ 0 & s_{42} & s_{43} & s_{44}\end{array}\right]$

A Monte Carlo analysis shows us that the population growth rate $\lambda$ is most sensitive to the germination rate $g$, summer survival of small plants $s$, and mortality due to floral herbivory $h$, see Tenhumberg et al. (2008). Using the values in Tenhumberg et al. (2008) for all variables except $g$, $h$, and $s$, we parameterize the matrix above and obtain

$$
\mathbf{A}=\left[\begin{array}{llll}
0 & 0 & 2043.80(1-h)(1-g) & 9289.98(1-h)(1-g) \\
.015 \mathrm{~s} & 0 & 1052.37 g(1-h) s & 4783.51 g(1-h) s \\
0 & .12 & .11 & 0 \\
0 & .02 & .27 & .17
\end{array}\right]
$$

With the nominal values $g=0.2142, s=0.516, h=0.942$, we obtain the nominal dominant eigenvalue $\lambda=1.538$. We are interested in values of parameters which yield asymptotic de- cline, i.e. by how much we need to perturb these nominal values to get the thistle population under control.

We want to use Theorem 2.2. First note that the individual rows in a matrix can be isolated into the form $\mathrm{de}^{T}$ where, if considering the first row, $\mathbf{d}=\left[\begin{array}{llll}1 & 0 & 0 & 0\end{array}\right]^{T}$ and $\mathbf{e}^{T}$ is the first row. In most single species models, removing the fecundity terms results in a substochastic matrix for all admissible parameters, yielding $r\left(\mathbf{A}_{0}\right)<1$. For example, replacing the first row of fecundities with zeros in a Leslie matrix results in a matrix with $r\left(\mathbf{A}_{0}\right)=0$ (see Appendix A) for all admissible parameters. Also in Appendix A, we show how to extract the fecundities from our matrix to obtain a substochastic matrix. For now, we use

$\mathbf{A}_{0}=\left[\begin{array}{llll}0 & 0 & 2043.80(1-h)(1-g) & 9289.98(1-h)(1-g) \\ 0 & 0 & 0 & 0 \\ 0 & .12 & .11 & 0 \\ 0 & .02 & .27 & .17\end{array}\right]$

then

$\mathbf{d}=\left[\begin{array}{l}0 \\ 1 \\ 0 \\ 0\end{array}\right]$

because we removed the second row, and

$\mathbf{e}^{T}=\left[\begin{array}{lll}.015 s & 0 & 1052.37 g(1-h) s \quad 4783.51 g(1-h) s\end{array}\right]$,

is the second row. Hence we have $\mathbf{A}=\mathbf{A}_{0}+\mathbf{d e}^{T}$ as required. To find the maximum $r\left(\mathbf{A}_{0}\right)$ for all admissible parameters, we use the results of Horn and Johnson (1985) who showed that if $\mathbf{A}>\mathbf{B}$ for non-negative matrices $\mathbf{A}$ and $\mathbf{B}$, then $r(\mathbf{A})>$ $r(\mathbf{B})$. Thus the maximum $r\left(\mathbf{A}_{0}\right)$ is found by substituting in the smaller limits for $h$ and $g$ (0.4 and 0.06; see Tenhumberg et al. (2008)). This yields a maximum $r\left(\mathbf{A}_{0}\right)$ of 0.17 . Clearly $\mathbf{d}$ is nonnegative, therefore, Theorem 2.2 applies to this system. It follows that the surface $C=\{(g, s, h) \in \mathcal{P} \mid \lambda(g, s, h)=1\}$ is the set $\Gamma$ of $(g, s, h)$ for which at least one eigenvalue of $\mathbf{A}$ is 1 . Intuitively, increases in survival and germination rate and a decrease in mortality due to floral herbivory increase the growth rate of the species. The surface $\Gamma$ (i.e. C), shown in Figure 1, gives us a partition of the parameter space; above the surface the population declines, and below the surface the population grows. The Matlab code to compute this $\lambda=1$ curve is available in Appendix D. For more information on this graphical method, see Hodgson and Townley (2004), Hodgson et al. (2006), and Deines et al. (2007).

Figure 1 also gives us an indication about how much we must change $(g, s, h)$, in order to bring the invasive population under control. The nominal values (black diamond in Figure 1), which give $\lambda=1.538$, appear to be a relatively large distance away from the surface $\Gamma$. There are many different possible norms in which we can use to measure this and often there is no a priori reason to chose one over the other. For illustrative purposes, we'll compare the 1-norm, 2-norm and $\infty$-norm where

$$
\begin{gathered}
\|\cdot\|_{1}=\left|v_{1}\right|+\ldots+\left|v_{S}\right|, \quad\|\cdot\|_{2}=\left(\left|v_{1}\right|^{2}+\ldots\left|v_{S}\right|^{2}\right)^{1 / 2} \text {, and } \\
\|\cdot\|_{\infty}=\max _{1 \leq i \leq S}\left|v_{i}\right|
\end{gathered}
$$

Since $\|\cdot\|_{\infty} \leq\|\cdot\|_{2} \leq\|\cdot\|_{1}$, the $\infty$-norm will yield a point which is "closer" to the growth-decline boundary than the point obtained via the 1-norm. In this example, the shortest distance to the surface from the nominal value in the 1-norm is .0406, in the 2-norm is .0404 and in the $\infty$-norm is .0363. The location of this closest point on the surface is given by the vectors $\langle .2142$, $.5159, .9825\rangle,\langle .2111, .5143, .9822\rangle$, and $\langle .1809, .4813, .9783\rangle$ for the 1-norm, 2-norm, and $\infty$-norm respectively. (The Matlab code for the 2-norm calculations is given in Appendix D; 


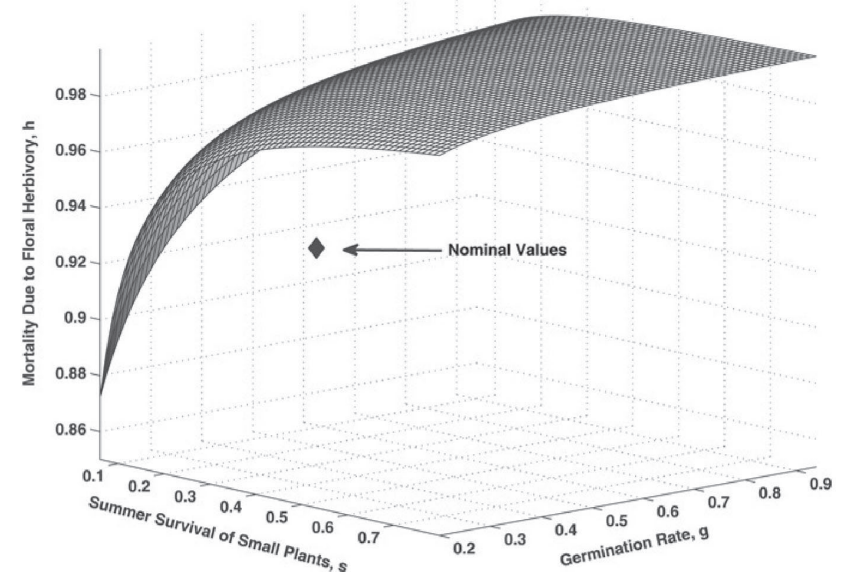

Figure 1. The surface $\lambda(g, s, h)=1$ in parameter space.

the code can be modified for more parameters and/or different norms.) Thus, in the Euclidian norm, the shortest distance in the parameter space is obtained by simultaneously changing $g$ to .2113 , changing $s$ to .5139 , and changing $h$ to .9822 . The goal is to move the nominal point to the other "side" of the $(\lambda=1)$-hypersurface as far as resources allow. In this particular example, to move the nominal point to the closest point on the $(\lambda=1)$-hypersurface for all these norms necessitates an increase in the mortality due to herbivory, $h$. This may involve the costly introduction of floral herbivore as biological control agents, which may not be a viable option for many population managers. To incorporate the different costs associated with changing the different parameters, a weighted norm could be used (Trefethen and Bau, 1997).

For this example, a better approach from a management standpoint would be to consider managing the summer survival of all plants (small, medium, and large) by the use of herbicides. Letting $x$ be the percentage increase in plant mortality due to herbicide application, the survivorships $c, d$, and $e$ in Equation (3.1) become $c(1-x), d(1-x)$, and $e(1-x)$. We can again apply Theorem 2.2 to help identify the growthdecline boundary. Figure 2 shows $h$ as a function of $x$ on the $\lambda(x, h)=1$ curve. Given the uncertainty in the mortality due to herbivory, $h$, a value for the percentage increase in plant mortality can be chosen to make the management decision robust in light of this uncertainty. For example, an $80 \%$ increase in the mortality of all plants over the course of a summer moves the nominal values (black diamond) in both Figures 2 $\& 3$ to the far right (black square) and on the other side of the $\lambda(x, h)=1$ curve. As shown in Figure 2, this gives decay for all $h>0.16$. The further away the black square is from the $\lambda(x, h)=$ 1 curve, the more robust this management decision is, regardless of the norm used. Figure 3 includes germination rate, $g$, as an uncertain variable. Again, increasing the mortality due to herbicides to $80 \%$ moves the nominal values (black diamond) to the black square on other side of the $\lambda(x, g, s)=1$ surface.

\subsection{An IPM thistle model}

We will illustrate our methods with a model, given in Ellner and Rees (2006), for the thistle O. illyricum, which we can write in the form (2.4). We will consider the effect that three of the parameters in the fecundity kernel have on $\lambda(\mathbf{A})$.

The population distribution of $O$. illyricum at time $t, n(x$, $a, t)$, is a function of the plant size $x$, and the age of the plant a. The plant size is a continuous variable taking on values between 0 and $M_{s^{\prime}}$ the maximum size, while the age is a discrete

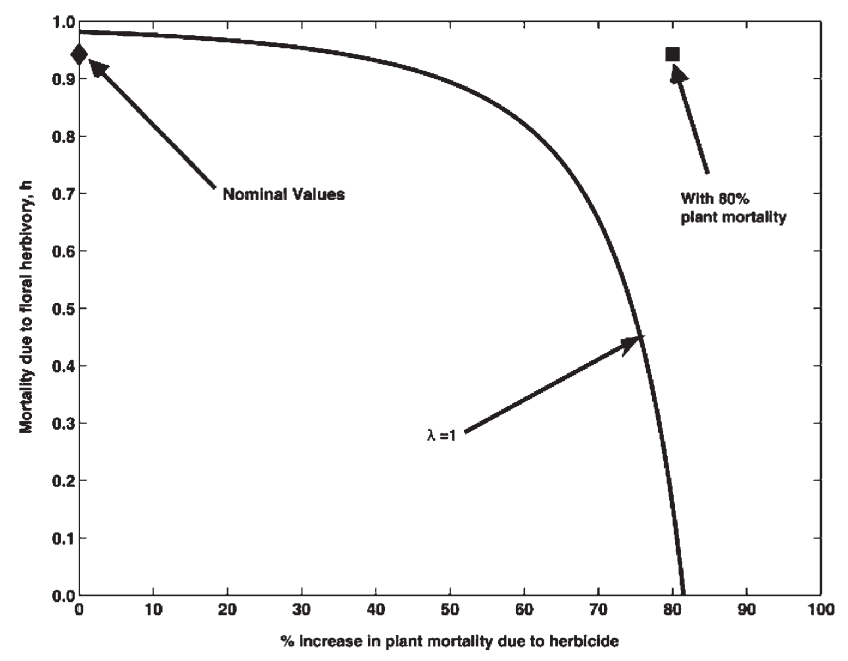

Figure 2 . The $\lambda(x, h)=1$ curve, where $x$ is the mortality of all summer plants, and $h$ is the mortality due to floral herbivory. The black diamond represents nominal values, the black square represents an $80 \%$ increase in mortality of the summer survival of all plants (small, medium, and large) due to management actions (e.g. application of herbicide).

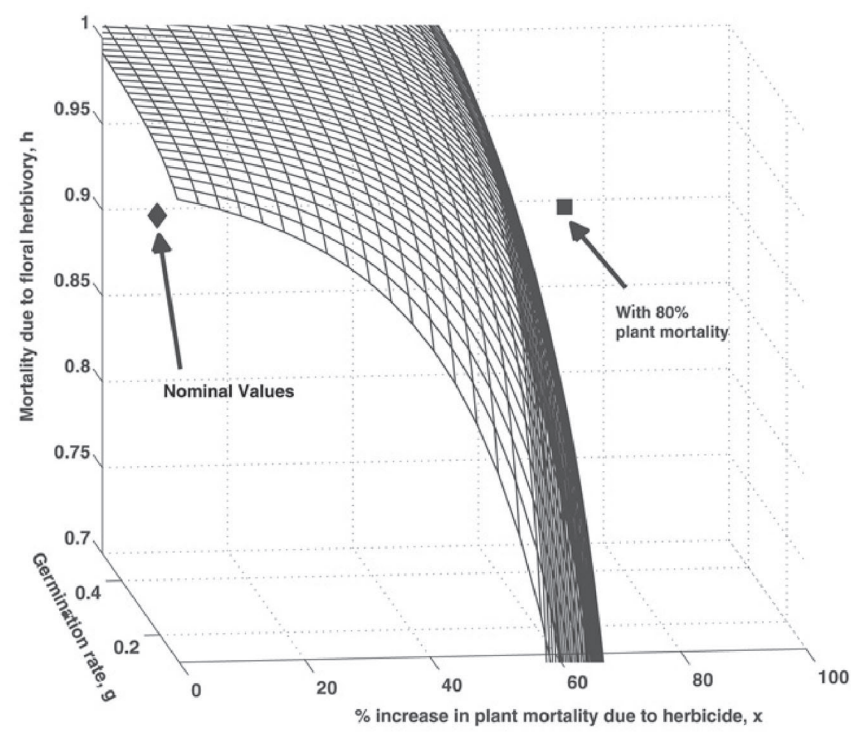

Fig. 3. The $\lambda(x, g, s)=1$ surface where $x$ is the mortality of all summer plants, $h$ is the mortality due to floral herbivory, and $g$ is the germination rate. The black diamond represents nominal values, the black square represents an $80 \%$ increase in mortality of the summer survival of all plants (small, medium, and large) due to management actions (e.g. application of herbicide).

variable which can take on values in $\left\{0,1, \ldots, M_{a}\right\}$, where $M_{a}$ is the maximum age. Let $\Omega=\left\{(x, a) \mid x \in\left[0, M_{s}\right], a \in\left\{0,1, \ldots, M_{a}\right\}\right\}$, and let $d(x, a)$ denote the product measure on $\Omega$ with Lebesgue measure in $x$ and discrete measure in $a$ (i.e. integrate over $x$ and sum over values of $a$ ). The kernel $k$ in Equation (2.4) can be written $k=p+f$, where $p$ is the "growth and survivorship kernel" and $f$ is the "fecundity kernel." The kernel describes how to get from state $(x, a)$ to state $(y, b)$, hence is a function of $(y, x, b, a)$. The population model can be written as $\mathbf{n}(t+1)=\mathbf{A n}(t)$ : here $\mathbf{A}$ is the operator defined on the Banach space

$$
\begin{aligned}
& \mathcal{B}=L_{1}(\Omega)=\left\{v(\cdot, \cdot)\left|\int_{0}^{M_{S}}\right| v(x, b) \mid d x<\infty\right. \\
& \text { for all } \left.b=0,1,2, \ldots, M_{a}\right\}
\end{aligned}
$$


given by

$$
(\mathbf{A v})(y, b)=\int_{\Omega}(p(y, x, b, a)+f(y, x, b, a)) v(x, a) d(x, a) .
$$

Expanding the product measure, (3.5) becomes

$$
(\operatorname{Av})(y, b)=\sum_{a=0}^{M_{a}} \int_{0}^{M_{s}}(p(y, x, b, a)+f(y, x, b, a)) v(x, a) d x .
$$

The population vector $\mathbf{n}(t)=n(\cdot, \cdot, t)$ is a vector in $\mathcal{B}$ for each $t$. The growth and survivorship kernel $p(y, x, b)$ is given by

$$
p(y, x, b, a)=s(x, a)\left[1-p_{f}(x, a)\right] g(y, x) \delta_{a, b-1}\left(1-\delta_{b, 0}\right),
$$

with component terms as follows: $s(x, a)>0$ is the yearly survivorship of a plant of size $x$ and age $a$ into a plant of size $x$ and age $a+1$; the Kronecker delta function $\delta_{i j}$ equals 1 if $i=$ $j$, else it equals 0 ; the growth of a plant from size $x$ to size $y$ is given by $g(y, x)>0$; and $p_{f}(x, a)$ is the probability of the plant of size $x$ and age $a$ to flower. Since O. illyricum is a monocarpic perennial, flowering results in death. Consequently $1-p_{f}(x, a)>0$ reflects the probability of the plant not flowering. The term $\delta_{a, b-1}=1$ when $a=b-1$, i.e. when plants are moving from one age class into the next. This prevents plants from aging more than one year at a time and also prevents them from getting younger with time. Since plants do not grow and survive into age $0,1-\delta_{b, 0}$ prevents this possibility. (For simplicity, these functions were not included in the original model in Ellner and Rees (2006).) Hence the growth and survival operator $\mathbf{A}_{0}$ is given by

$$
\begin{aligned}
\left(\mathbf{A}_{0} \mathbf{v}\right)(y, b)= & \int_{\Omega} s(x, a)\left[1-p_{f}(x, a)\right] g(y, x) \delta_{a, b-1} \\
& \times\left(1-\delta_{b, 0}\right) v(x, a) d(x, a) .
\end{aligned}
$$

The fecundity kernel $f(y, x, b, a)$ contains three underlying parameters, $p_{e^{\prime}} p_{1}$, and $p_{2^{\prime}}$, whose uncertainty can have a broad impact on the asymptotic population growth rate. Here, $p_{e}$ is the probability of seedling establishment and $f_{n}(x)=e^{p_{1}+p_{2} x}$ is the number of seeds per adult of size $x$. Since the model assumes that the state of the offspring is independent of the parents, new plants are distributed into size classes by the probability distribution $\varphi(y)>0$. The kernel $f$ is therefore given by

$$
f(y, x, b, a)=s(x, a) p_{f}(x, a) p_{e} e^{p_{1}+p_{2} x} \varphi(y) \delta_{b, 0} .
$$

The total number of offspring produced by the population distribution at time $t$ is

$$
\left(\mathbf{e}^{T} \mathbf{n}\right)(t)=\int_{\Omega} s(x, a) p_{f}(x, a) p_{e} e^{p_{1}+p_{2} x} n(x, a, t) d(x, a)
$$

These offspring are then distributed into size classes described by the vector $\mathbf{d} \in \mathcal{B}$ given by

$$
\mathbf{d}=\varphi(\cdot) \delta_{b, 0}
$$

that is, $\mathbf{d}$ is represented by the function $d(y, b)$ which is $\varphi(y)$ when $b=0$ and is 0 otherwise. Thus the population distribution at time $t+1$ is

$$
\mathbf{n}(t+1)=\mathbf{A}(\mathbf{n}(t))=\left(\mathbf{A}_{0}+\mathbf{d e} \mathbf{e}^{T}\right)(\mathbf{n}(t)) .
$$

We need to verify that this system satisfies the condition in Theorem 2.5. The cone $K$ used in Theorem B.6 in Appendix B is the set of all positive functions $v(x, a)$ on $\Omega$. The operator $\mathbf{A}_{0}: \mathcal{B} \rightarrow \mathcal{B}$ is nilpotent, i.e. there is a positive integer $m$ such that $\mathbf{A}_{0}^{m}=0$. This is because $\mathbf{A}_{0}$ advances the age of the population distribution, and in the absence of any population input the population will die off in finite time. Nilpotent operators are well-known to have spectral radius 0 , i.e. $r\left(\mathbf{A}_{0}\right)=0$. The vector $\mathbf{d} \in \mathcal{B}$, and the operators $\mathbf{e}^{T}: \mathcal{B} \rightarrow \mathbb{R}, \mathbf{A}_{0}$, and $\mathbf{A}$ are clearly positive. The kernel $k(y, x, b, a)=p(y, x, b, a)+f(y, x, b$, $a)$ is bounded on a bounded set $\left[0, M_{s}\right] \otimes\left\{0,1, \ldots, M_{a}\right\}$, so by the Hilbert-Schmidt theorem $\mathbf{A}$ is compact (see Bachman and Narici (1966)). It is proved in Ellner and Rees (2006) that A is $u$-bounded. Therefore, this system satisfies the hypotheses of Theorem 2.2 with $r\left(\mathbf{A}_{0}\right)=0$, so we can conclude that any positive eigenvalue of $\mathbf{A}$ is in fact the leading eigenvalue. $\mathbf{A}$ satisfies the conditions of Theorem 2.2 for any positive $p_{e^{\prime}} p_{1^{\prime}}$, and $p_{2}$. In addition, since $p_{e}$ is a probability, the set, $\mathcal{P}$, of admissible parameters is $\left\{\left(p_{e^{\prime}} p_{1}, p_{2}\right) \mid p_{e} \in[0,1], p_{1}>0, p_{2}>0\right\}$.

Remark 3.1. The kernel $k$ in the model given in Ellner and Rees (2006) also depends on the quality $q$, which represents the variability between plants and is assumed to be constant throughout each plant's lifespan. To add this dependence to the model here would only require including a distribution function $\beta(q)$ to the operator $\mathbf{d}$ which distributes the newborns into quality classes much like $\varphi(y)$ distributes the newborns into size classes.

The probability of seedling establishment, $p_{e^{\prime}}$ cannot be calculated accurately due to the presence of a seed bank, so we expect substantial uncertainty in the value of this parameter. Suppose $p_{e}$ has a nominal value of 0.025 . (Note that while in Ellner and Rees (2006), $p_{e}=0.025$, they use the value 0.03 in their computer code.) We take the nominal value of the fecundity intercept, $p_{1}$, to be -11.84 , and the nominal value of the fecundity slope, $p_{2}$, to be 2.27 . Both of these calculated numbers have the following standard errors as given in Ellner and Rees (2006): 4.43 for the fecundity intercept and 0.60 for the fecundity slope. Thus the seed production per plant has a wide range of possibilities.

We first consider the effect of $p_{e}$ and $p_{1}$ on $\lambda(\mathbf{A})$, which is an easy case, since both variables can be pulled out of the integral defining $\mathbf{e}^{T}$. In particular, we can write

$$
\mathbf{e}^{T}=p_{e} e^{p}{ }_{1} \mathbf{e}_{p}^{T}, \text { where } \mathbf{e}_{p}^{T} \mathbf{v}=\int_{\Omega} e^{p}{ }_{2}^{x} p_{f}(x, a) s(x, a) v(x, a) d(x, a) .
$$

For now we assume that $p_{2}$ is fixed at its nominal value.

Theorem 3.2. Suppose that $\lambda>0$ is an eigenvalue of $\mathbf{A}$. Then

$$
p_{e}=(\lambda / \gamma) e^{-p_{1}}
$$

where

$$
r=\mathbf{e}_{p}^{T}\left(\mathbf{I}+\frac{\mathbf{A}_{0}}{\lambda}+\frac{\mathbf{A}_{0}^{2}}{\lambda}+\ldots+\frac{\mathbf{A}_{0}^{m-1}}{\lambda}\right) \varphi
$$

The proof of this is given in Appendix B. Note that $\gamma$ is a real number which can be approximated numerically. If there is a particular asymptotic growth rate $\lambda>0$ which is desired, Theorem 3.2 gives a formula for the curve of $\left(p_{1}, p_{e}\right)$ values which lead to $\lambda$.

Figure 4 shows the curves $\left\{\left(p_{1}, p_{e}\right) \mid \lambda\left(p_{1}, p_{e}\right)=\alpha\right\}$ for $\alpha$ increasing from 0.70 to 1.3 in 0.1 increments. The nominal point computed in Ellner and Rees (2006) is (-11.84, 0.025), which leads to $\lambda=0.9878$. We can see from the figure that $\lambda$ varies considerably more with larger uncertainties in the fecundity intercept $p_{1}$ than with large uncertainties in the probability for seedling establishment $p_{e}$. The graphs shows the range of $p_{1}$ within its standard error of the nominal value. In this range the variation in $\lambda$ includes both values signifying dramatic asymptotic decay and values signifying dramatic asymptotic growth. Thus we do not consider $\lambda$ to be robust with respect to large changes in $p_{1}$. Since the probability of seedling establishment was not measured directly (see Ellner and Rees (2006)), it is unclear whether $\lambda$ is robust to changes in $p_{e}$ within the unknown standard error. If $p_{e}$ remains within $25 \%$ of its current values, i.e. between 0.019 and 0.031 and if the fecundity inter- 


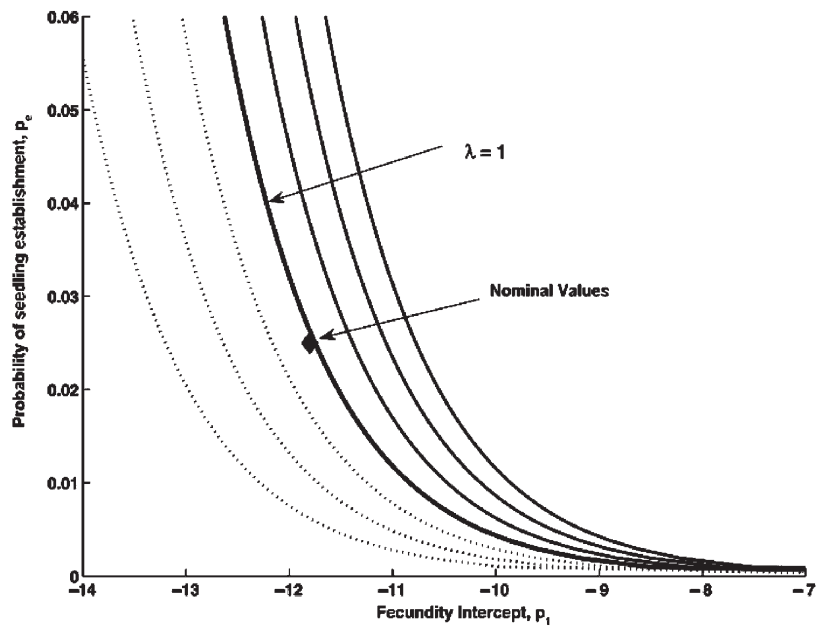

Figure 4. The curves show values of $\left(p_{1}, p_{e}\right)$ for which $\lambda=.70, .80, .90$, $1.0,1.1,1.2$, decreasing in value to the left of the $\lambda=1$ curve and increasing in value to the right. The black diamond marks the nominal point $(-11.84,0.025)$.

cept remains fixed at its nominal value, $-11.84, \lambda$ varies by less than 0.10 . Yet if $p_{e}$ increases by as much as $50 \%$ to 0.375 , then $\lambda$ increases by approximately 0.10 . Likewise, if $p_{e}$ decreases by $50 \%$ to 0.0125 , then $\lambda$ decreases by almost 0.20 . Hence $\lambda$ is more robust to increases in $p_{e}$ than to decreases to $p_{e}$.

We now consider simultaneous variation in the fecundity intercept $p_{1}$, fecundity slope $p_{2}$, and probability of seedling establishment $p_{e^{\text {. }}}$ This is more difficult computationally, since $p_{2}$ cannot be pulled out of the integral defining $\mathbf{e}^{T}$. We use the same derivation as above, but now $\gamma$ in Equation (3.13) is a function $r\left(p_{2}\right)$, which needs to be calculated for every $p_{2}$. We can solve for $p_{e}$ in terms of $p_{1}$ and $r\left(p_{2}\right)$. Figure 5 shows the surface $\left\{\left(p_{e^{\prime}} p_{1}, p_{2}\right) \mid \lambda\left(p_{e^{\prime}} p_{1}, p_{2}\right)=1\right\}$ for a range of admissible $\left(p_{e^{\prime}}\right.$ $\left.p_{1}, p_{2}\right)$. This surface divides the $\left(p_{e^{\prime}} p_{1}, p_{2}\right)$ parameter space into those parameters above the surface, which lead to asymptotically increasing population, and those parameters below the surface, which lead to asymptotic decreasing population. Notice that as $p_{1}$ and $p_{2}$ decrease within the standard error, then $p_{e}$ must increase exponentially to maintain $\lambda\left(p_{e^{\prime}} p_{1^{\prime}}, p_{2}\right)=1$, and eventually increases above 1 , an impossibility for a probability. Figure 6 shows the $\lambda=1$ contours for $p_{e}=.01, .03, .10$, .30, .60, and 1.0. The black diamond marks the nominal values of $\left(p_{1}\right.$, $\left.p_{2}\right)=(-11.84,2.27)$. On the graph, one can see that if $p_{1}$ and/or $p_{2}$ are increased slightly, $p_{e}$ needs only to decrease slightly in order to maintain $\lambda=1$. On the other hand, if $p_{1}$ and/or $p_{2}$ are decreased slightly, $p_{e}$ must increase to a much greater value in order to maintain $\lambda=1$. Thus our graph shows $\lambda$ is not robust at all to decreases in $p_{1}$ and $p_{2}$ and to increases in $p_{e}$.

\section{Discussion}

The asymptotic growth rates of populations are parameterized by vital rates. These vital rates are summarized in a projection model (PM) (in this paper either an IPM or PPM), while the asymptotic growth rate is given by the PM's dominant eigenvalue. This leads us to study the dependence of dominant eigenvalues in PMs on these vital rates. Perhaps the simplest parameterized PM is the Leslie matrix $\mathbf{L}$ of a population with $S$ age classes. Here, the parameters consist of fecundity values $f_{1}, \ldots, f_{S}$ in the top row and survival probabilities $\sigma_{1}, \ldots$, $\sigma_{S-1}$ in a sub-diagonal. The Leslie matrix $\mathbf{L}$ has only one positive real eigenvalue. Cushing and Yicang (1994) showed that the asymptotic growth rate of an age-structured population,

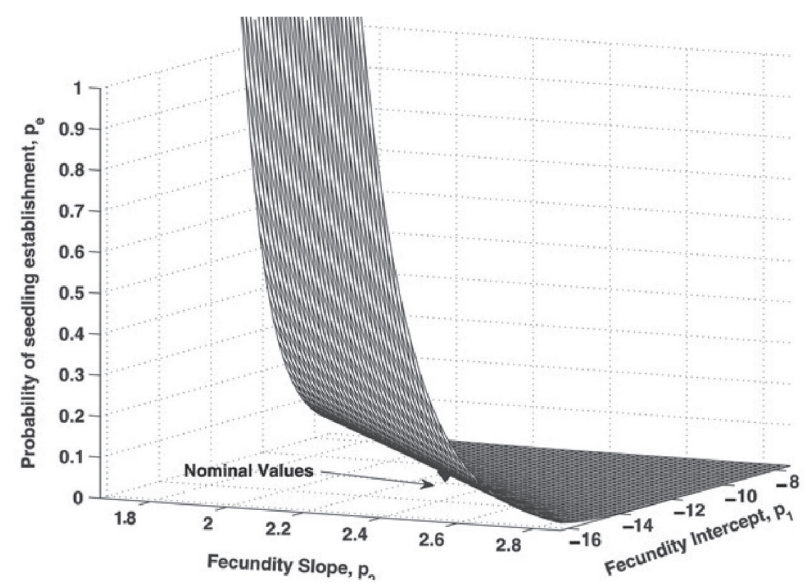

Figure 5. The parameter space of fecundity intercept $p_{1}$, fecundity slope $p_{2}$, and probability of seedling establishment $p_{e}$ for $\lambda=1$.

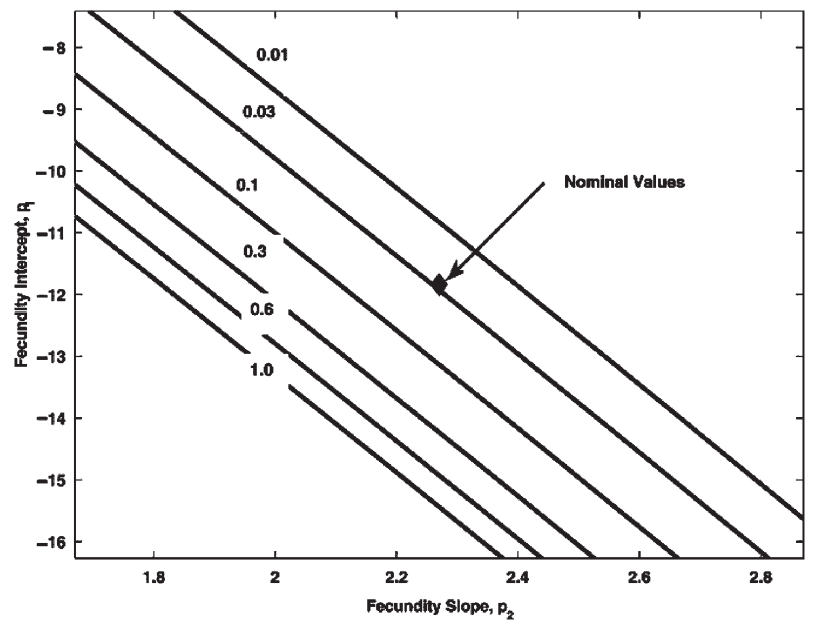

Figure 6 . The contours are the $\lambda=1$ curves for various values of the probability of seedling establishment $p_{e}$. The nominal values for the fecundity intercept and fecundity slope are indicated by the black diamond.

i.e. the leading eigenvalue of $\mathbf{L}$, is greater than one if, and only if, the reproductive value, $f_{1}+f_{2} \sigma_{1}+f_{3}\left(\sigma_{1} \sigma_{2}\right)+\ldots+f_{s}\left(\sigma_{1} \ldots \sigma_{s-1}\right)$ is greater than one. Hence the relationship

$$
f_{1}+f_{2} \sigma_{1}+f_{3}\left(\sigma_{1} \sigma_{2}\right)+\ldots+f_{s}\left(\sigma_{1} \ldots \sigma_{s-1}\right)=1
$$

characterizes the boundary between growing and declining age-structured populations. Cushing and Yicang (1994) also calculated the net reproductive value for other types of population projection matrices in terms of the matrix entries. These results yield a growth-decline boundary based on the calculation for net reproductive value. In addition, given the reproductive value $R$, they showed that if $R<1$, then $R \leq \lambda \leq 1$ and if $R>1$, then $1 \leq \lambda \leq R$. However, their results do not lead to a robustness analysis of $\lambda$ with respect to the nominal vital rates.

Suppose we are identifying the growth-decline boundary, but we do not have an easy way of determining whether an eigenvalue of $\mathbf{A}$ is the leading eigenvalue. This is not particularly difficult if the nominal population is declining. This is because all of the eigenvalues of the nominal model are less than 1 , and so to seek parameter values yielding leading eigenvalues greater than one reduces to finding parameter values so that one eigenvalue hits 1 . The same applies at more general growth-decline boundaries $\lambda=\rho$. Thus, the robustness of population decline is an "easy" problem. 
If, however, we start with a base point given by a nominal growing population, which already has at least one eigenvalue greater than 1, then the boundary is no longer determined simply by parameter values achieving an eigenvalue of 1 . This is because we cannot guarantee a priori that this eigenvalue at 1 is the leading eigenvalue. We see that robustness of population growth is more subtle than robustness of population decline, and is simplified if we can easily identify when an eigenvalue is the leading eigenvalue.

We identify a class of PM's for which the growth-decline boundary $\lambda=\rho$ is given precisely by those parameters for which $\rho$ is an eigenvalue. This means we can determine the growth-decline boundary simply by evaluating the parameterized characteristic polynomial evaluated at $\rho$. We must assume a decomposition of the parameterized matrix in terms of a matrix $\mathbf{A}_{0}$ (typically a survival matrix), whose dominant eigenvalue we know (usually by inspection) to be less than $\rho$, and a rank one perturbation matrix. The existence of such a decomposition very often follows immediately from the construction of the PM, and is ecologically natural. For instance, for Leslie matrices $\mathbf{A}_{0}$ is obtained by zeroing out the fecundities so that $\mathbf{A}_{0}$ has dominant eigenvalue $\lambda=0$. In fact, any PPM or IPM which satisfies our assumptions, whose population advances to a new age class at every time step until it reaches some maximum age and dies, will have nilpotent $\mathbf{A}_{0}$, so $r\left(\mathbf{A}_{0}\right)=0$. This is true for a Leslie matrix and in our IPM example. Thus, we get the familiar result that any PM which can be written in the general form of $\mathbf{A}=\mathbf{A}_{0}+\mathbf{d e}^{T}$, with nilpotent $\mathbf{A}_{0}$, will have only one positive eigenvalue.

Having identified the growth-decline boundary in such analytically simple terms, we can then explore how "close" the nominal values are to the boundary as we vary one, two or even all parameters. While the closest point on the boundary depends on the norm chosen, in our PPM example there was not much difference between using the Euclidean norm and the 1-norm.

Additionally, the growth-decline boundary shows the interconnectedness between all of the parameters. A management action increasing one life-history parameter value may have the benefit of moving the nominal point far enough on the other "side" of the $(\lambda=1)$-hypersurface that the uncertainties in the other parameters are no longer a major concern. In such a case population growth (or decline) is robust, even with the uncertainties in the other parameters.

While our method does not explicitly address stochastically varying environments, it does allow for analysis of spatially and temporally varying environments when this variation is within some tolerance level. For example, in environments which vary slightly, the stochastic contribution to the variance of the parameters can be treated like a parameter uncertainty. Our methods then can be applied in the same way as we did in the PPM thistle example, where the data uncertainty represents spatial variation. If the environments vary considerably, such as in the case of hurricanes, fires or other catastrophes, then our method would not give useful information since the parameter variation is too large. Our methods also do not take into consideration possible density-dependence.

Despite these shortcomings mentioned in the previous paragraph, this method generates a clear growth-decline boundary for PPMs and IPMs and points conservation managers towards a strategy which can be robust in the face of the uncertainty in the parameters.

Acknowledgments - This work was supported by NSF REU Site Grant 0354008. RR was supported in part by NSF Grant 0606857. ST was supported in part by a Leverhulme Trust Research Fellowship.
We'd also like to thank the two anonymous reviewers for Theoretical Population Biology for their helpful comments.

\section{Appendix A. PPM theoretical results}

Proof of Theorem 2.2. We first assume that $\mathbf{d}$ is nonnegative. Suppose $\lambda>r\left(\mathbf{A}_{0}\right)$ is an eigenvalue of $\mathbf{A}$. Then for some nonzero $\mathbf{v} \in \mathbb{R}^{n}$,

$$
\left(\mathbf{A}_{0}+\mathbf{d e}^{T}\right) \mathbf{v}=\lambda \mathbf{v}
$$

Since $\lambda$ is not an eigenvalue of $\mathbf{A}_{0}$, we can re-arrange (A.1) to give:

$$
\mathbf{v}=\left(\lambda \mathbf{I}-\mathbf{A}_{0}\right)^{-1} \mathbf{d e}^{T} \mathbf{v}
$$

Note that if $\mathbf{e}^{T} \mathbf{v}=0$, then Equation (A.2) implies that $\mathbf{v}=0$, which is a contradiction. Hence $\mathbf{e}^{T} \mathbf{v}$ or $-\mathbf{e}^{T} \mathbf{v}$ is a positive scalar. Without loss of generality assume that $\mathbf{e}^{T} \mathbf{v}$ is positive, since if $\mathbf{e}^{T} \mathbf{v}$ is not positive, we can replace $\mathbf{v}$ by $-\mathbf{v}$. Since $\lambda>$ $r\left(\mathbf{A}_{0}\right)$, we can expand $\left(\lambda \mathbf{I}-\mathbf{A}_{0}\right)^{-1}$ in a power series:

$$
\left(\lambda \mathbf{I}-\mathbf{A}_{0}\right)^{-1}=\frac{1}{\lambda}\left(\mathbf{I}+\frac{\mathbf{A}_{0}}{\lambda}+\frac{\mathbf{A}_{0}^{2}}{\lambda^{2}}+\ldots\right) .
$$

Since $\mathbf{A}_{0}^{j}$ is nonnegative for $j=0,1, \ldots$, it follows that $\left(\lambda \mathbf{I}-\mathbf{A}_{0}\right)^{-1} \mathbf{d}$ is nonnegative. Then Equation (A.2) shows that $\mathbf{v}$ is also nonnegative. Since $\mathbf{A}$ is primitive, there is an integer $k$ such that $\mathbf{A}^{k}$ $>0$, i.e. every entry of $\mathbf{A}^{k}$ is positive. Since

$$
\mathbf{v}=\frac{1}{\lambda^{k}} \mathbf{A}^{k} \mathbf{v}
$$

we see that $\mathbf{v}>0$. Hence $\lambda$ is a positive eigenvalue of the primitive matrix A with a strictly positive eigenvector $\mathbf{v}$. The Perron-Frobenius now implies that $\lambda=r(\mathbf{A})=\lambda(\mathbf{A})$.

If $\mathbf{d}$ is not nonnegative but $\mathbf{e}^{T}$ is nonnegative, we can apply the result to the transpose $\mathbf{A}^{T}$ of $\mathbf{A}$. Then the result follows immediately from the facts that $\mathbf{A}^{T}=\mathbf{A}_{\mathbf{0}}^{T}+\mathbf{e d}^{T}, r\left(\mathbf{A}_{\mathbf{0}}^{T}\right)=r\left(\mathbf{A}_{0}\right)$, and $\lambda\left(\mathbf{A}^{T}\right)=\lambda(\mathbf{A})$.

Corollary A.1. A primitive Leslie matrix can have only one positive eigenvalue.

Proof. A Leslie matrix is of the form

$$
\mathbf{A}=\left[\begin{array}{ccccc}
f_{1} & f_{2} & \ldots & f_{S-1} & f_{S} \\
\sigma_{1} & 0 & \ldots & 0 & 0 \\
0 & \ddots & 0 & \ldots & 0 \\
\vdots & \ddots & \ddots & \ddots & \vdots \\
0 & \ldots & \ldots & \sigma_{S-1} & 0
\end{array}\right]
$$

This can be written in the form $\mathbf{A}_{0}+\mathbf{d e}^{T}$, with $\mathbf{d}=\left[\begin{array}{llll}1 & 0 & \ldots & 0\end{array}\right]^{T}$ $\geq 0, \mathbf{e}^{T}=\left[f_{1}, \ldots, f_{N}\right] \geq 0$. The survival matrix $\mathbf{A}_{0}$ is a lower triangular matrix with a zero diagonal. Such a matrix has 0 as it's only eigenvalue, so $r\left(\mathbf{A}_{0}\right)=0$. Hence Theorem 2.2 shows that $\mathbf{A}$ has only one eigenvalue larger than $r\left(\mathbf{A}_{0}\right)=0$.

- 0 -

As another example of Theorem 2.2, the matrix (3.1) is of the general 4 by 4 form given in (3.2). We can write

$$
\mathbf{A}=\mathbf{A}_{0}+\mathbf{d e}^{T}=\left[\begin{array}{llll}
0 & 0 & 0 & 0 \\
s_{21} & 0 & 0 & 0 \\
0 & s_{32} & s_{33} & 0 \\
0 & s_{42} & s_{43} & s_{44}
\end{array}\right]+\left[\begin{array}{l}
c_{1} \\
c_{2} \\
0 \\
0
\end{array}\right]\left[\begin{array}{llll}
0 & 0 & f_{3} & f_{4}
\end{array}\right]
$$

When the $s_{i, j}$ are survival parameters, each column in $\mathbf{A}_{0}$ sums to less than 1, so $\mathbf{A}_{0}$ is substochastic and $r\left(\mathbf{A}_{0}\right)<1$ for all admissible parameters when $f_{3}$ and $f_{4}$ are fecundities, $\mathbf{e}^{T}>0$. Therefore PPMs of this form satisfy the hypotheses in Theorem 2.2. 


\section{Appendix B. IPM theoretical results}

Let $\mathcal{B}$ be a Banach space, and $\mathbf{A}: \mathcal{B} \rightarrow \mathcal{B}$ be a linear operator. The integral operators of the form (2.4) we consider in this paper are all bounded operators. If the function $k(\cdot, \cdot)$ is continuous and the set $\Omega$ is bounded-which is typical when $\Omega$ is the set of stages a population can take-then $\mathbf{A}$ is also a compact operator, see for instance Bachman and Narici (1966). One characterization of a compact operator is that it can be uniformly approximated by finite rank operators, so reliable numerical results can be obtained for these systems. A consequence of compactness is that the only non-zero spectrum $\mathbf{A}$ has are eigenvalues. In order for an integral equation of the form (2.4) to satisfy the conclusions of Proposition 2.1, we need to make further assumptions about A. Fortunately, these assumptions are natural for most single-species models. To this end, we need a few definitions and previous results. The following definitions can be found in many sources, including Krasnosel'skij et al. (1989), Zabreyko et al. (1975), and Ellner and Rees (2006).

In order to work with "positive operators" and "nonnegative operators" the same way we work with positive and nonnegative matrices, we first need a partial order " $\geq$ " on $\mathcal{B}$.

Definition B.1. Let $\mathcal{B}$ be a Banach space. A cone is a nonempty, closed, convex subset, $K \subseteq \mathcal{B}$ provided two conditions hold:

1. If $x \in K$ and $\alpha \geq 0$ then $\alpha x \in K$.

2. If $x,-x \in K$ then $x=0$.

Definition B.2. A cone $K$ induces a partial order on the Banach space $\mathcal{B}$, denoted by " $\geq$ " (or resp. " $\leq$ ") where $x \geq y$ (or resp. $x \leq y$ ) for $x, y \in \mathcal{B}$ means $x-y \in K$ (or resp. $y-x \in K$ ). If $x>y$ (or resp. $x<y$ ), we mean that $x \geq y$ and $x \neq y$ (or resp. $x \leq y$ and $x \neq y)$.

Definition B.3. A cone is called reproducing if $K \ominus K=\mathcal{B}$, that is, every element in the Banach space can be written as the sum of one element in $K$ plus the negative of another element in $K$.

When $\mathcal{B}=L_{1}(\Omega)$, we typically use the cone $K$ of functions which are nonnegative on $\Omega$, which is reproducing. Hence if $f, g \in L_{1}(\Omega)$, then $f \leq g$ means $g-f$ is a nonnegative function on $\Omega$, i.e. $g-f$ is in the cone $K$. When $=\mathbb{R}^{n}$, we use the cone of vectors which have nonnegative components.

Definition B.4. Let $K_{1}$ and $K_{2}$ be cones in Banach spaces $\mathcal{B}_{1}$ and $\mathcal{B}_{2}$ respectively. A bounded linear operator, $\mathbf{A}$, is called positive if it maps $K_{1}$ into the cone $K_{2}$, i.e. $A K_{1} \subseteq K_{2}$.

Definition B.5. Let $u \in K, u \neq 0$. A positive linear operator, $\mathbf{A}$, is called $u$-bounded if for each $x \in K$ there exists constants $\alpha=$ $\alpha(x)>0$ and $\beta=\beta(x)>0$ such that $\alpha u \leq A x \leq \beta u$.

Roughly speaking, an integral projection model is $u$ bounded if the distribution of offspring states is independent of the state of the parent. Let

$$
k^{(j+1)}(y, x)=\int_{\Omega} k(y, z) k^{(j)}(z, x) d z .
$$

We say that the kernel $k$ is power positive if there exists some integer $j>0$ such that $k^{(j)}(y, x)>0$ for all $x, y \in \Omega$. It is shown in Ellner and Rees (2006) that if $k$ is power positive and continuous and the domain is compact, then $\mathbf{A}$ is $u$-bounded.

The following theorem is a generalization of the PerronFrobenius theorem for matrices to compact, $u$-bounded operators. It is proved in Krasnosel'skij et al. (1989).
Theorem B.6. Let $\mathcal{B}$ be a Banach space with a reproducing cone $K$ which determines a partial order. Let $\mathbf{A}$ be a positive, compact, ubounded linear operator on $\mathcal{B}$. Then

1. $r(\mathbf{A})$ is an eigenvalue whose corresponding eigenvector is an element of $K$. This eigenvalue is the spectral radius of $\mathbf{A}$.

2. This eigenvalue is simple and its corresponding eigenvector is the unique (up to normalization) eigenvector in $\mathrm{K}$.

3. Every other eigenvalue of $\mathbf{A}$ is less in magnitude than $\lambda$.

The following follows as in the matrix case.

Corollary B.7. Let $\mathcal{B}$ be a Banach space with a reproducing cone $K$ which determines a partial order. Let $\mathbf{A}$ be a positive, compact, $u$-bounded linear operator on $\mathcal{B}$. Then the conclusions of Proposition 2.1 hold.

Proof of Theorem 2.5. We first assume that $\mathbf{d}$ is positive. Suppose $\lambda>r\left(\mathbf{A}_{0}\right)$ is an eigenvalue of $\mathbf{A}$. Then for some nonzero $\mathbf{v} \in \mathcal{B}$,

$$
\left(\mathbf{A}_{0}+\mathbf{d e}^{T}\right) \mathbf{v}=\lambda \mathbf{v}
$$

Since $\lambda$ is not an eigenvalue of $\mathbf{A}_{0}$, we can re-arrange (B.2) to give:

$$
\mathbf{v}=\left(\lambda \mathbf{I}-\mathbf{A}_{0}\right)^{-1} \mathbf{d e}^{T} \mathbf{v} .
$$

Note that if $\mathbf{e}^{T} \mathbf{v}=0$, then (B.3) implies that $\mathbf{v}=0$, which is a contradiction. Hence $\mathbf{e}^{T} \mathbf{v}$ or $-\mathbf{e}^{T} \mathbf{v}$ is a positive scalar. Without loss of generality assume that $\mathbf{e}^{T} \mathbf{v}$ is positive, since if $\mathbf{e}^{T} \mathbf{v}$ is not positive, we can replace $\mathbf{v}$ by $\mathbf{- v}$. Since, we can expand $\left(\lambda \mathbf{I}-\mathbf{A}_{0}\right)^{-1}$ in a power series:

$$
\left(\lambda \mathbf{I}-\mathbf{A}_{0}\right)^{-1}=\frac{1}{\lambda}\left(\mathbf{I}+\frac{\mathbf{A}_{0}}{\lambda}+\frac{\mathbf{A}_{0}^{2}}{\lambda^{2}}+\ldots\right) .
$$

Since $\mathbf{A}_{0}$ is positive and $\mathbf{d}$ is positive, it follows that $\mathbf{A}_{0}{ }^{k} \mathbf{d}$ is positive for all $k \geq 0$. Hence it follows from (B.4) that $\left(\lambda \mathbf{I}-\mathbf{A}_{0}\right)^{-1} \mathbf{d}$ is positive. Then (B.3) shows that $\mathbf{v}$ is also positive, i.e. it is in the cone. Hence $\lambda$ is a positive eigenvalue of $\mathbf{A}$ with associated positive eigenvector $\mathbf{v}$. Hence by Theorem B.6, $\lambda$ must be the leading eigenvalue of $\mathbf{A}$, so $\lambda=\lambda(\mathbf{A})$. Since $\mathbf{A}$ is compact, its nonzero spectrum consists only of eigenvalues, so $\lambda(\mathbf{A})=r(\mathbf{A})$.

If $\mathbf{d}$ is not nonnegative but $\mathbf{e}^{T}$ is nonnegative, we can apply the result to the adjoint $\mathbf{A}^{*}$ of $\mathbf{A}$. We denote the adjoint of $\mathbf{d}$ by $\mathbf{d}^{T}$ and the adjoint of $\mathbf{e}^{T}$ by $\mathbf{e}$. Then the result follows immediately from the facts that $\mathbf{A}^{*}=\mathbf{A}_{0}^{*}+\mathbf{e d}^{T}, r\left(\mathbf{A}_{0}^{*}\right)=r\left(\mathbf{A}_{0}\right)$, and $\lambda\left(\mathbf{A}^{*}\right)=\lambda(\mathbf{A})$.

- 0

Proof of Theorem 3.2. Suppose that $\lambda>0$ is an eigenvalue of $\mathbf{A}$ with eigenvector $\eta \in \mathcal{B}$. Then

$$
\left(\mathbf{A}_{0}+\mathbf{d e}^{T}\right) \eta=\lambda \eta
$$

Let $\mathbf{I}: \mathcal{B} \rightarrow \mathcal{B}$ be the identity operator, and note that $\left(\lambda \mathbf{I}-\mathbf{A}_{0}\right)^{-1}$ is a bounded operator since $\mathbf{A}_{0}$ is a compact operator with $r\left(\mathbf{A}_{0}\right)=0$. After some algebraic manipulation,

$$
\left(\lambda \mathbf{I}-\mathbf{A}_{0}\right)^{-1} \mathbf{d e} \mathbf{e}^{T} \eta=\eta
$$

which implies that

$$
\mathbf{e}^{T}\left(\lambda \mathbf{I}-\mathbf{A}_{0}\right)^{-1} \mathbf{d e}^{T} \eta=\mathbf{e}^{T} \eta
$$

If the scalar $\mathbf{e}^{T} \eta=0$, then (B.5) implies that $\lambda$ is an eigenvalue of $\mathbf{A}_{0}$, which is not possible. Hence $\mathbf{e}^{T} \eta$ is a nonzero scalar and we can divide (B.6) by $\mathbf{e}^{T} \eta$ to obtain

$$
\mathbf{e}^{T}\left(\mathbf{I}-\mathbf{A}_{0} / \lambda\right)^{-1} \mathbf{d}(1)=\lambda
$$




$$
\text { Since } \begin{aligned}
\mathbf{A}_{0}^{m} & =0, \\
\lambda & =p_{e} e^{p_{1}} \mathbf{e}_{p}^{T}\left(\mathbf{I}-\mathbf{A}_{0} / \lambda\right)^{-1} \mathbf{d}(1) \\
& =p_{e} e^{p_{1}} \mathbf{e}_{p}^{T}\left(\mathbf{I}+\mathbf{A}_{0} / \lambda+\mathbf{A}_{0}^{2} / \lambda+\ldots+\mathbf{A}_{0}^{m-1} / \lambda\right) \mathbf{d}(1) .
\end{aligned}
$$

Note that $\mathbf{d}(1)=\varphi$ to get (3.13). Therefore we can solve for $p_{e}$ in terms of $p_{1}$ to get (3.12).

๑ 0 -

\section{Appendix C. The side of a hypersurface}

The set of admissible parameters $\mathcal{P}$ can be decomposed into $C \cup C_{+} \cup C_{-}$, where these sets are defined in (2.5), (2.6), and (2.7). The next Lemma shows that $\mathcal{P}-C=C_{+} \cup C_{-}$, a disconnected subset of $\mathcal{P}$ with $C_{+}$separated from $C_{-}$, which is a precise way of saying that $C_{+}$is on one "side" of $C$ and $C_{-}$is on the other "side" of $C$.

Lemma C.1. Suppose that $\mathbf{A}\left(p_{1}, p_{2}, \ldots, p_{m}\right)$ is continuous in the operator norm for $\left(p_{1}, p_{2}, \ldots, p_{m}\right) \in \mathbb{R}$. Let $\left(p_{1}, p_{2^{\prime}}, \ldots, p_{m}\right)$ and $\left(q_{1}\right.$, $\left.q_{2}, \ldots, q_{m}\right)$ be in $\mathcal{P}$ with $\lambda\left(\left(p_{1}, p_{2}, \ldots, p_{m}\right)\right)>\lambda_{0}$ and $\lambda\left(\left(q_{1}, q_{2}, \ldots\right.\right.$, $\left.\left.q_{m}\right)\right)<\lambda_{0}$. Let $\gamma:[0,1] \rightarrow \mathcal{P}$ be any continuous function with $\gamma(0)$ $=\left(p_{1}, p_{2}, \ldots, p_{m}\right)$ and $\gamma(1)=\left(q_{1}, q_{2}, \ldots, q_{m}\right)$. Then there exists $t_{0} \in$ $[0,1]$ with $\lambda\left(\gamma\left(t_{0}\right)\right)=\lambda_{0}$.

Proof. Since the codomain of $\gamma$ is $\mathcal{P}$, we have that $\lambda(\gamma(t)) \subset \mathbb{R}$. Since $\mathbf{A}\left(p_{1}, p_{2}, \ldots, p_{m}\right)$ is continuous in the operator norm, $\lambda\left(p_{1}\right.$, $p_{2}, \ldots, p_{m}$ ) is continuous (Kato, 1980). Using the continuity of $\gamma$, the intermediate value theorem states that we must have some $t_{0}$ with $\lambda\left(\gamma\left(t_{0}\right)\right)=\lambda_{0}$. 口 ם $\mathbf{0}$

In all of our examples the sets are connected, but we note that this Lemma does not however guarantee that $C_{,} C_{+}$, and $C_{-}$are connected in general.

\section{Appendix D. Numerical calculations}

\section{D.1. Plotting the $(\lambda=1)$-hypersurface}

In our first program, we used Matlab's symbolic toolbox (Matlab, 2007) for plotting the $(\lambda=1)$-hypersurface. A second method, as shown by the second computer program below, does not require the use of Matlab's symbolic toolbox and is specific to our example. For more information on this method, please see Hodgson and Townley (2004), Hodgson et al. (2006) and Deines et al. (2007).

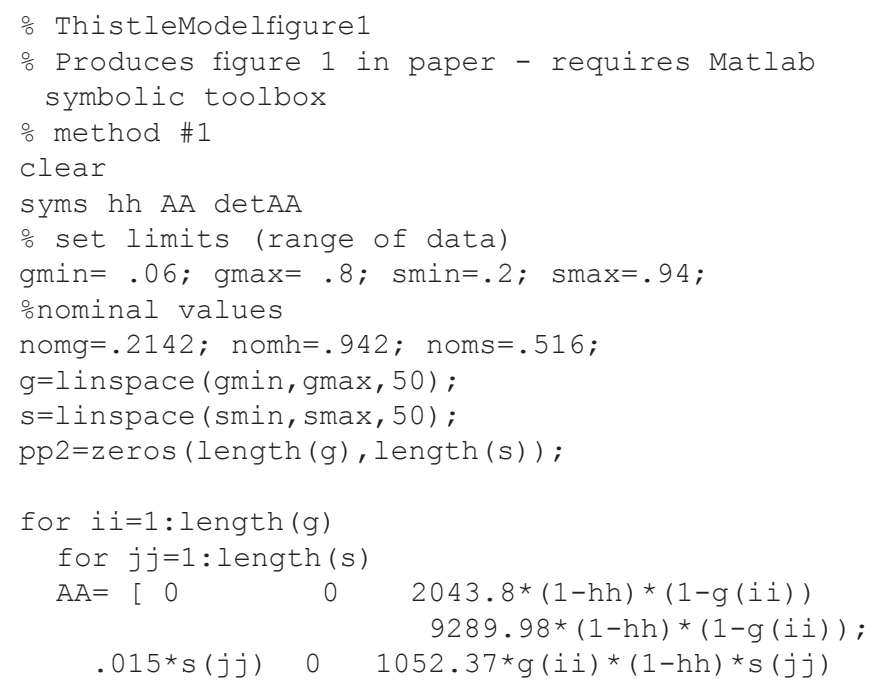

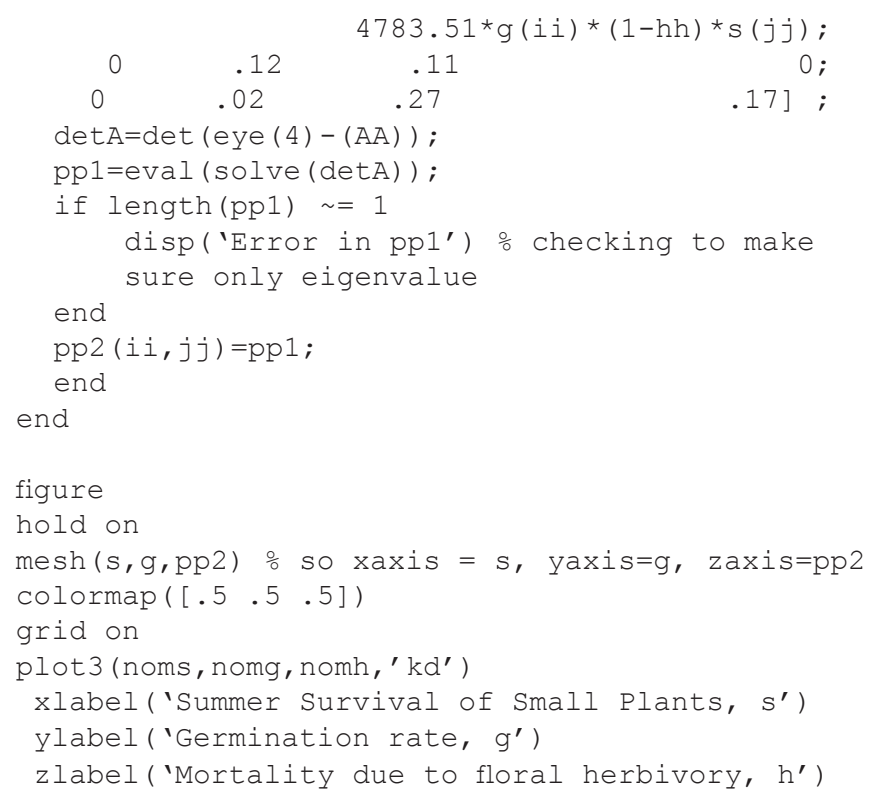

In the second method, we plot $\lambda(s, g, h)=1$, where $s$ is the summer survival of small plants, $g$ is the the germination rate, and $h$ is the mortality due to floral herbivory, writing $s$ as a function of $g$ and $h$. First, we rewrite $\mathbf{A}$ as $\mathbf{A}=\mathbf{A}_{0}+s \mathbf{d} \tilde{\mathbf{e}}^{T}$ where $\mathbf{A}_{0}$ and $\mathbf{d}$ are given by Equations (3.3) and (3.4) respectively and

$$
\tilde{\mathbf{e}}^{T}=\left[\begin{array}{lll}
.015 & 0 & 1052.37 g(1-h) \\
4783.51 g(1-h)
\end{array}\right]
$$

Since $\lambda=1$ is not an eigenvalue of $\mathbf{A}_{0}$, we see that $\lambda$ is an eigenvalue of $\mathbf{A}$ with eigenvector $\mathbf{v}$ if and only if $s \tilde{\mathbf{e}}^{T}\left(\mathbf{I}-\mathbf{A}_{0}\right)^{-1} \mathbf{d}$ $=1\left(\right.$ Hodgson and Townley, 2004). Since $\tilde{\mathbf{e}}^{T}\left(\mathbf{I}-\mathbf{A}_{0}\right)^{-1} \mathbf{d}$ is a scalar, then

$$
s=\left[\tilde{\mathbf{e}}^{T}\left(\mathbf{I}-\mathbf{A}_{0}\right)^{-1} \mathbf{d}\right]^{-1}
$$

This gives $s$ as a function of $g$ and $h$ when $\lambda=1$. Hence the $(\lambda=$ 1)-hypersurface can be plotted as follows:

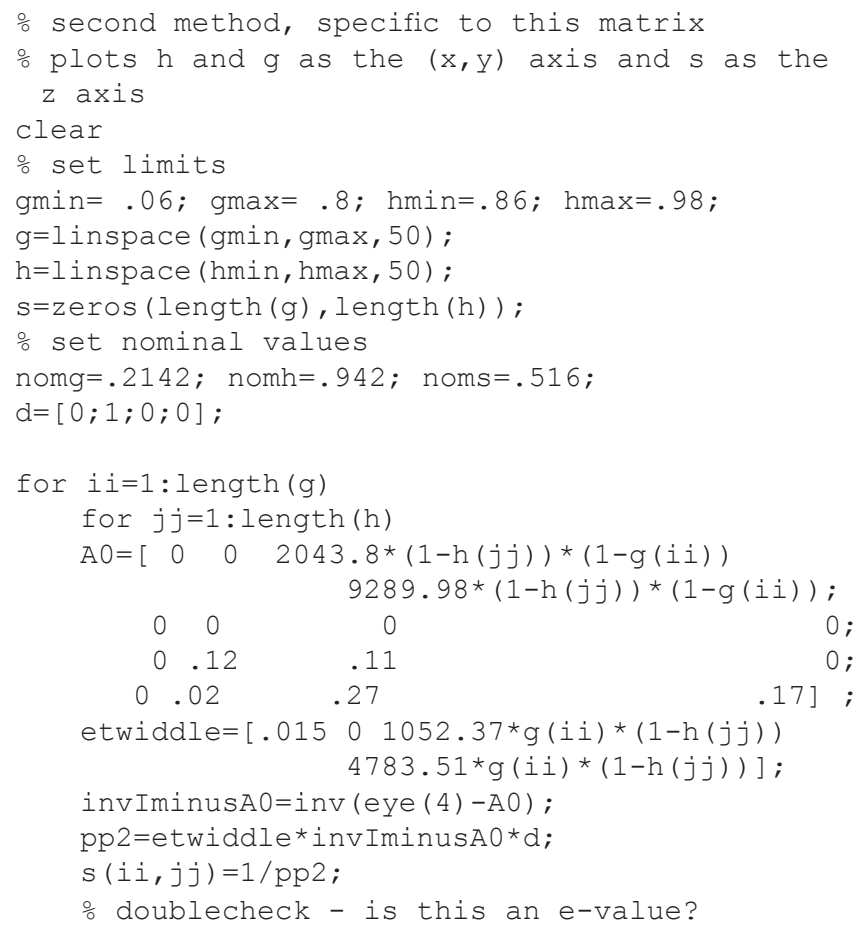




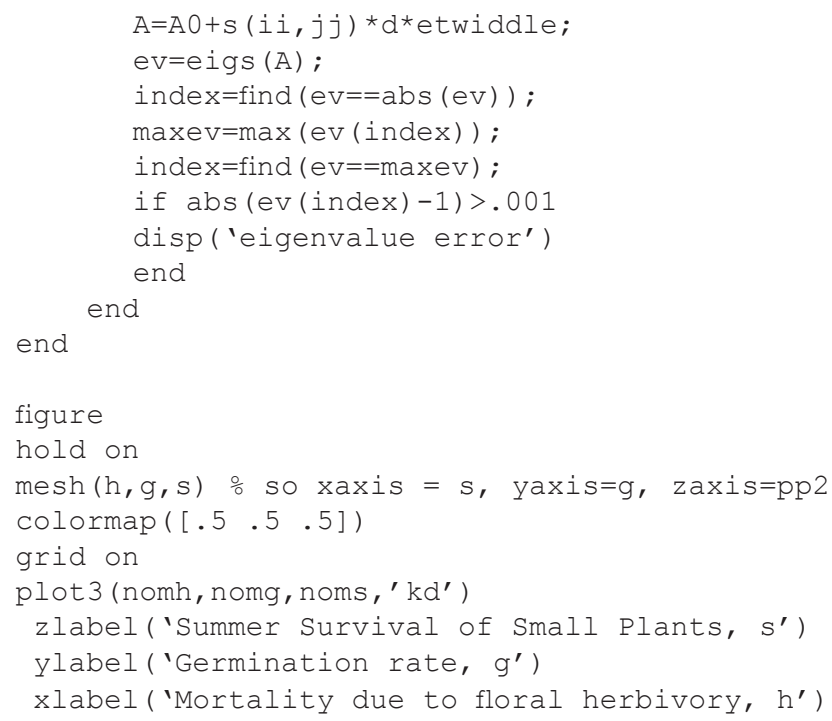

\section{D.2. Determining the closest point on the $(\lambda=1)$-hypersurface}

To determine the closest point on the $(\lambda=1)$-hypersurface requires solving a constrained, nonlinear multivariable problem. Let $x=(g, h, s)$ be a point on the $(\lambda=1)$-hypersurface and let $x_{\text {nom }}=\left(g_{\text {nom }}, h_{\text {nom }}, s_{\text {nom }}\right)$ be the nominal values for $g, h$, and $s$ respectively. Let $L$ and $U$ be the lower bound vector and upper bound vector for $x$, respectively. Using the same derivation in the above subsection, for $x=(g, h, s)$ to be a point on the $(\lambda$ $=1$ )-hypersurface, Equation (D.2) needs to be satisfied. Therefore, we are finding the minimum of a problem defined by

$\min _{x}\left\|x-x_{n o m}\right\|_{2}$ such that $\left\{\begin{array}{c}L \leq x \leq U, \\ \left|s \tilde{\mathbf{e}}^{T}\left(\mathbf{I}-\mathbf{A}_{0}\right)^{-1} \mathbf{d}-1\right|-s_{\text {tol }} \leq 0,\end{array}\right.$

where $\mathbf{A}_{0}, \mathbf{d}$, and $\tilde{\mathbf{e}}^{T}$, are given in Equations (3.3), (3.4), and (D.1) respectively. Because strict equalities are impossible when computing $s$, we have set a tolerance level, $s_{t o l}$, for the accuracy of this calculation.

A brute force method of calculating the shortest distance from the nominal point to the hypersurface can also be used. This requires dividing the hypersurface up into a grid and individually calculating the distance from the nominal point to each point in the grid; then one checks for the minimum over all grid points.

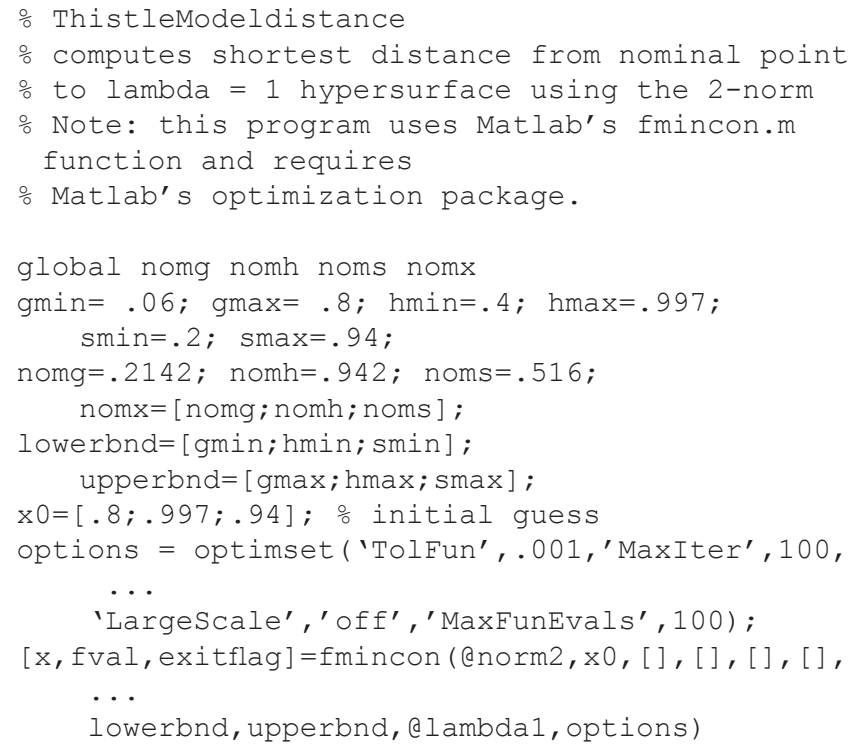

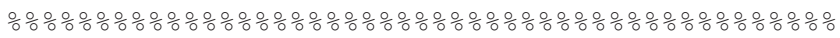

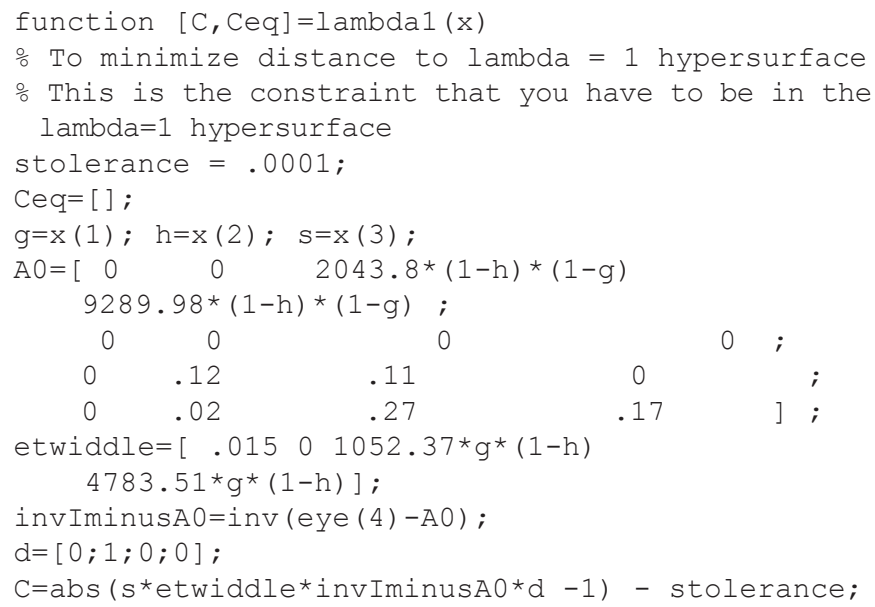

\section{References}

Bachman and Narici, 1966 G. Bachman and L. Narici, Functional Analysis, Academic Press, New York (1966).

Briese et al., $1994<$ D. T. Briese, A. W. Sheppard, H. Zwölfer, and P. E. Boldt, Structure of the phytophagous insect fauna of Onopordum thistles in the northern Mediterranean basin, Biological Journal of the Linnean Society 53 (1994), pp. 231-253.

Briese et al., $2002 \varangle$ D. T. Briese, W. J. Pettit, A. Swirepik, and A. Walker, A strategy for the biological control of Onopordum spp. thistles in south-eastern Australia, Biocontrol Science and Technology 12 (2002), pp. 121-136.

Caswell, $2000 \varangle \mathrm{H}$. Caswell, Prospective and retrospective perturbation analysis: Their roles in conservation biology, Ecology 81 (2000), pp. 619-627.

Caswell, 2001 H. Caswell, Matrix Population Models, Construction, Analysis, and Interpretation (2nd ed. ), Sinauer Associates, Inc., Sunderland, MA (2001).

Cushing and Yicang, $1994<$ J. M. Cushing and Z. Yicang, The net reproductive value and stability in matrix population models, Natural Resource Modeling 8 (1994), pp. 297-333.

Deines et al., 2007 A. Deines, E. Peterson, D. Boeckner, J. Boyle, A. Keighley, J. Kogut, J. Lubben, R. Rebarber, R. Ryan, B. Tenhumberg, S. Townley, and A. J. Tyre, Robust population management under uncertainty for structured population models, Ecological Applications 17 (2007), pp. 2175-2183.

de Kroon et al., $2000 \varangle \mathrm{H}$. de Kroon, J. van Groenendael, and J. Ehrlén, Elasticities: A review of methods of model limitations, Ecology 81 (2000), pp. 607-618.

Doak et al., $2005<$ D. F. Doak, K. Gross, and W. F. Morris, Understanding and predicting the effects of sparse data on demographic analyses, Ecology 86 (2005), pp. 1154-1163.

Easterling, 1998 ४ M. R. Easterling, The integral projection model: Theory, analysis and application. Ph. D. diss. North Carolina State University, Raleigh. 1998.

Easterling et al., $2000 \varangle$ M. R. Easterling, S. P. Ellner, and P. M. Dixon, Size-specific sensitivity: Applying a new structured population model, Ecology 81 (2000), pp. 694-708. 
Ehrlén, $2000 \varangle \mathrm{J}$. Ehrlén, The dynamics of plant populations: Does the history of individuals matter?, Ecology 81 (2000), pp. 1675-1684.

Ehrlén and van Groenendael, 1998 \. Ehrlén and J. van Groenendael, Direct perturbation analysis for better conservation, Conservation Biology 12 (1998), pp. 470-474.

Ellner and Rees, $2006 \varangle$ S. P. Ellner and M. Rees, Integral projection models for species with complex demography, American Naturalist 167 (2006), pp. 410-428.

Goss, $1924 \varangle$ W. L. Goss, The vitality of buried seeds, Journal of Agricultural Research 29 (1924), pp. 349-362.

Groves et al., $1990<$ R. H. Groves, J. J. Burdon, and P. E. Kaye, Demography and genetics of Onopordum in southern New South Wales, Journal of Ecology 78 (1990), pp. 47-55.

Guretzky and Louda, 1997 J. A. Guretzky and S. M. Louda, Evidence for natural biological control: Insects decrease survival and growth of a native thistle, Ecological Applications 7 (1997), pp. 1330-1340.

Hodgson and Townley, $2004<$ D. J. Hodgson and S. Townley, Linking management changes to population dynamic responses: The transfer function of a projection matrix perturbation, Journal of Applied Ecology 41 (2004), pp. 1155-1161.

Hodgson et al., 2006 D. J. Hodgson, S. Townley, and D. McCarthy, Robustness: Predicting the effects of life history perturbations on stage-structured population dynamics, Theoretical Population Biology 70 (2006), pp. 214-224; doi: 10.1016/j. tpb.2006.03.004

Horn and Johnson, $1985<$ R. A. Horn and C. R. Johnson, Matrix Analysis, Cambridge University Press, Cambridge (1985).

Kato, 19804 T. Kato, Perturbation Theory for Linear Operators, Springer, New York (1980).

Krasnosel'skij et al., $1989 \varangle \mathrm{M}$. A. Krasnosel'skij, Je. A. Lifshits, and A. V. Sobolev, Positive Linear Systems - The Method of Positive Operators, Heldermann Verlag, Berlin, Germany (1989).

Louda and Rand, 2003 S. M. Louda and T. A. Rand, Native thistles: Expendable or integral to ecosystem resistance to invasion?. In: P. Kareiva and S. A. Levin, Editors, The Importance of Species: Perspectives on Expendability and Triage, Princeton University Press, Princeton (2003), pp. 5-15.
Lubben et al., $2008<$ J. Lubben, B. Tenhumberg, A. Tyre, and R. Rebarber, Management recommendations based on matrix projection models: The importance of considering biological limits, Biological Conservation 141 (2008), pp. 517-523; doi: 10.1016/j.biocon.2007.11.003

Mandujano et al., $2001<\mathrm{M}$. C. Mandujano, C. Montaña, M. Franco, J. Golubov, and A. Flores-Martínez, Integration of demographic annual variability in a clonal desert cactus, Ecology 82 (2001), pp. 344-359.

Matlab, 2007 Matlab R2007a, 2007. The MathWorks, Inc., Natick, MA.

Mills et al., 1999 L. S. Mills, D. F. Doak, and M. J. Wisdom, Reliability of conservation actions based on elasticity analysis of matrix models, Conservation Biology 13 (1999), pp. 815-829.

Pettit et al., $1996<$ W. J. Pettit, D. T. Briese, and A. Walker, Aspects of thistles population dynamics with reference to Onopordum, Plant Protection Quarterly 11 (1996), pp. 232-235.

Seno and Nakajima, 1999 H. Seno and H. Nakajima, Transition matrix model for persistence of monocarpic perennial plant population under periodically ecological disturbance, Ecological Modelling 117 (1999), pp. 65-80; doi: 10.1016/ S0304-3800(99)00010-1

Tenhumberg et al., $2008 \varangle$ B. Tenhumberg, S. M. Louda, J. O. Eckberg, and M. Takahashi, Monte Carlo analysis of parameter uncertainty in matrix models of the weed Cirsium vulgare, Journal of Applied Ecology 45 (2008), pp. 438-447.

Trefethen and Bau, $1997<$ L. N. Trefethen and D. Bau III, Numerical Linear Algebra, Society for Industrial and Applied Mathematics, Philadelphia (1997).

van Tienderen, 19954 P. H. van Tienderen, Life cycle trade-offs in matrix population models, Ecology 76 (1995), pp. 2482-2489.

Wisdom and Mills, 19974 M. J. Wisdom and L. S. Mills, Sensitivity analysis to guide population recovery: Prairie-chickens as an example, Journal of Wildlife Management 61 (1997), pp. 302-312.

Zabreyko et al., 1975 P. P. Zabreyko, A. I. Koshelev, M. A. Krasnosel'skii, S. G. Mikhlin, L. S. Rakovshchik, and V. Ya. Stet'senko, Integral Equations - A Reference Text, Noordhoff International, Leyden, The Netherlands (1975). 